\title{
EFFECTS OF HETEROGENEITY AND GLOBAL DYNAMICS OF WEAKLY CONNECTED SUBPOPULATIONS
}

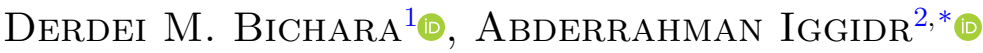 \\ AND SOUAD YACHEUR ${ }^{2,3}$
}

\begin{abstract}
We develop a method that completely characterizes the global dynamics of models with multiple subpopulations that are weakly interconnected. The method is applied on two classes of models with multiple subpopulations: an epidemic model that involves multiple host species and multiple vector species and a patchy vector-borne model. The method consists of two main steps: reducing the system using tools of large scale systems and studying the dynamics of an auxiliary system related the original system. The developed method determines the underlying dynamics and the "weight" of each subpopulations with respect to the dynamics of the whole population, and how the topology of the connectivity matrix alters the dynamics of the overall population. The method provides global stability results for all types of equilibria, namely trivial, boundary or interior equilibria.
\end{abstract}

Mathematics Subject Classification. 92D30, 34D23, 34D20, 34D40, 34A34.

Received December 15, 2019. Accepted June 6, 2021.

\section{INTRODUCTION}

Mathematical models in population dynamics that incorporate age, group, or spatial heterogeneities use network to describe the interactions between the units of the model. The overall dynamics of these models is, naturally, driven by the interconnectedness of the sub-populations. For instance, the effects of mixing and connectivity patterns of populations on the spread of infectious diseases have long been recognized in literature $[1,26,27]$. The complete or partial dynamics of these types of models have been investigated in $[3-5,8,10,11$, 19, 20, 24, 29, 34], and the references therein. However, a common point in these papers is that, a rigorous and complete mathematical analysis of these models requires the subpopulations to be strongly connected. That is, an irreducibility hypothesis on the network configuration is assumed in these studies [8, 9, 11, 12, 23, 29]. This hypothesis synchronizes the dynamics in each of the involved subpopulation or patch. For instance, for epidemic models, typically the disease dies out in all subpopulations, when the basic reproduction number is less than one, and persists in all of them otherwise.

However, in most cases the graph that represents the connections between the subpopulations is not strongly connected and the dynamics is not well-understood. Although it has long been assumed in the literature of

Keywords and phrases: Large scale systems, network configuration, weakly connected populations, global stability, Lyapunov functions.

${ }^{1}$ Department of Mathematics, California State University, Fullerton, CA 92831, USA.

2 Université de Lorraine, CNRS, Inria, IECL, 57000 Metz, France.

3 Department of Mathematics, University of Tlemcen, Tlemcen 13000, Algeria.

* Corresponding author: abderrahman.iggidr@inria.fr 
ecological and epidemiological models of dispersal, that when the connectivity (between patches or groups) is reducible, the dynamics in these patches or groups will be somewhat independent, a clear mathematical formalism has been lacking. It has been pointed out in [2] the possibility to have mixed equilibria, with some patches disease free and others with persisting disease when the patches are weakly connected. This has been formalized in [16]. In this paper, we propose a method that gives a complete characterization of the dynamics of a class of mathematical models that captures the interactions between subpopulations, regardless of the topology of their connectivity matrix. Indeed, although the effects of mixing and connectivity patterns on the dynamics of populations have long been recognized in literature [1, 2, 21, 26, 27], the extend to which these impact the outcome is not well-understood. The method we provides answers to questions such as: What is the underlying dynamics of each subpopulations? What is the "weight" of each subpopulations with respect to the dynamics of the whole population? Which subpopulation is connected to which? And how the topology of the connectivity matrix alters the dynamics of the overall population?

As a template, we use the proposed method on investigating the dynamics of two of the most complex class of diseases with multiple subpopulations: zoonoses -infectious diseases caused by pathogens transmissible under natural conditions from vertebrate animals to humans and vector-borne diseases models in a patchy environment. Zoonoses represent $75 \%$ of emerging and re-emerging infectious diseases, of which $40 \%$ are vectorborne [25]. Moreover, a class of these zoonoses is multi-host and multi-vector. That is the pathogen shared by multiple definitive or intermediate host species and transmitted by multiple arthropod vector species. These multifaceted aspects challenge our ability to understand and control zoonoses [36], a class of diseases that cause over 20 billion USD of direct economic burden worldwide [25, 37]. A key factor in controlling these multi-host and multi-vector model is assessing the contribution of each host and vector species in the disease dynamics. Indeed, designing control programs for zoonoses that target only some host species (humans for instance) may lead to ineffective control effort [36]. Moreover, for many zoonoses, all hosts or vector species are not created equal [17] as different host and vector species differ in their propensity to infect or acquire infection due to their ecological habitats or behavior. Similarly, humans and arthropods are often heterogeneously distributed across space making the risk of malaria and other vector-borne diseases transmission highly variable [18, 31]. This high variability leads to some areas with stable transmission and others wit unstable transmission [18]. Moreover, the risks of transmission in areas or patches is not only tied to the clusters of mosquitoes and humans, but also on the mobility from and to these patches [29, 31]. When the patches are weakly connected, determining the sinks and sources of these vector-borne diseases allows informed intervention strategies that target the more prolific patches and therefore a cost-effective control strategies.

The choice of these two types of models is also motivated by the fact that the dynamics of these models have been well understood if the network is strongly connected $[8,11,24,29]$, a necessary step to determine the behavior of models with weakly connected subpopulations.

In this paper, we investigate the dynamics of these two classes of model when the network connectivity is not necessarily irreducible. Particularly, we propose a method that completely characterizes how host heterogeneity, vector heterogeneity, and connectivity patterns among subpopulations alter or mitigate disease dynamics. Although we illustrate our method using epidemic models with multiple subpopulations, the framework is applicable for all systems involving mixing of multiple subpopulations, including immunological and ecological models. The punch of our method consists of distinguishing which group or patch acts as sink or source of the infection, thereby providing a road-map for control strategies. Our framework also identifies the classes of populations that are futile to target for control, unless some other group of populations have been mitigated.

The paper is organized as follows: Section 2 presents the overall method using a general model. In Section 3, we illustrate the method on two classes of models: multi-host and multi-vector zoonoses models (Sect. 3.1) and vector-borne models in a patchy environments (Sect. 3.2). We briefly revisit the derivation of these two models and the basic properties before presenting the main result, which is the global stability of equilibria, regardless of the structure of the network configuration. These equilibria could be interior (all positive components) or mixed (nonnegative components). Section 4 provides an illustration of the method for a lower dimension system and Section 5 collects our concluding remarks. 


\section{MAIN RESUlT}

Many problems in population dynamics such as metapopulations, multi-groups can be written as:

$$
\left\{\begin{array}{l}
\dot{\boldsymbol{x}}_{1}=f_{1}\left(\boldsymbol{x}_{1}, \boldsymbol{x}_{2}, \ldots, \boldsymbol{x}_{n}\right) \\
\dot{\boldsymbol{x}}_{2}=f_{2}\left(\boldsymbol{x}_{1}, \boldsymbol{x}_{2}, \ldots, \boldsymbol{x}_{n}\right) \\
\vdots \\
\dot{\boldsymbol{x}}_{k}=f_{k}\left(\boldsymbol{x}_{1}, \boldsymbol{x}_{2}, \ldots, \boldsymbol{x}_{n}\right) \\
\vdots \\
\dot{\boldsymbol{x}}_{n}=f_{n}\left(\boldsymbol{x}_{1}, \boldsymbol{x}_{2}, \ldots, \boldsymbol{x}_{n}\right)
\end{array}\right.
$$

where $\boldsymbol{x}_{i}$ is the vector of each subpopulation and $f_{i}$ is the function that describes how the subpopulation evolves for itself and how it is connected with the other subpopulations. Many advances have been made in an attempts to understand the dynamics of these types of models in different settings. The dynamics of these models naturally depends on how these populations are interconnected. The global stability of steady states for these models has been partially or completely investigated $[5,8,9,11,14,19,20,23,24,27,29$, 30] when the matrix that represents the connectivity between the subpopulations, groups or patches is irreducible. Typically, if the irreducibility hypothesis holds, there are only two equilibria, namely the trivial equilibrium and the interior equilibrium and the trajectories either converge to the trivial equilibrium in all patches or converge towards the interior equilibrium in all of them depending on a threshold condition. The goal of this section is to provide a method that paves the way in understanding the global dynamics of models when the populations involved are weakly interconnected, if the dynamics is well-understood when the network is irreducible. Suppose that System (2.1), as a model that describes the evolution of multiple populations, is biologically grounded. That is, the trajectories of System (2.1) remain positive and bounded.

Let $M$ the matrix that describes the interactions between the subpopulations in (2.1). It is well known [6] that the matrix $M$ is irreducible if and only the corresponding directed graph is strongly connected. That leads to our main hypothesis:

H: The global dynamics of Model (2.1) is known if $M$ is irreducible.

If $M$ is reducible, the corresponding graph of the network connectivity is not strongly connected. However, it can be partitioned into connected components. Particularly, by a suitable permutation of the subpopulations, the matrix $M$ can take the form:

$$
M=\left(\begin{array}{cccc}
M_{11} & 0 & \ldots & 0 \\
M_{21} & M_{22} & \ldots & 0 \\
\vdots & \vdots & \ddots & \vdots \\
M_{s 1} & M_{s 2} & \ldots & M_{s s}
\end{array}\right)
$$

where each block $M_{i i}$ is square and is either irreducible or a $1 \times 1$ matrix. The graphs corresponding to these $M_{i i}$ are called irreducible components. Notice that some or all of the $M_{i j}$ with $i \neq j=2, \ldots, i-1$ can be equal to 0 . If $M_{i j}=0$, for all $i \neq j$, then the different irreducible components $M_{i i}$, for $1 \leq i \leq s$, are disconnected and, using $\mathbf{H}$, the global dynamics System (2.1) is straightforward. Now, suppose that some of the $M_{i j}$ with $i \neq j$ are non zeros.

Throughout the rest of this article, we will use the following notations. For two real matrices $A$ and $B$, we will write $A \leq B$ if $A(i, j) \leq B(i, j)$ for all $i$ and $j, A<B$ if $A \leq B$ and $A \neq B$, and $A \ll B$ if $A(i, j)<B(i, j)$ for all $i$ and $j$. In a similar way, we will use the same notations for real vectors. 
The key of the method consists of studying incrementally the dynamics of the model, starting with $M_{11}$. With the re-arranged graph, System (2.1) can be written as:

$$
\left\{\begin{array}{l}
\dot{\boldsymbol{x}}_{1}=f_{1}\left(\boldsymbol{x}_{1}\right) \\
\dot{\boldsymbol{x}}_{2}=f_{2}\left(\boldsymbol{x}_{1}, \boldsymbol{x}_{2}\right) \\
\vdots \\
\dot{\boldsymbol{x}}_{k}=f_{k}\left(\boldsymbol{x}_{1}, \boldsymbol{x}_{2}, \ldots, \boldsymbol{x}_{k}\right) \\
\vdots \\
\dot{\boldsymbol{x}}_{s}=f_{s}\left(\boldsymbol{x}_{1}, \boldsymbol{x}_{2}, \ldots, \boldsymbol{x}_{s}\right),
\end{array}\right.
$$

where the $\boldsymbol{x}_{i}$ are of appropriate dimensions. Notice the dynamics of $\boldsymbol{x}_{1}$ is independent of $\boldsymbol{x}_{2}, \boldsymbol{x}_{3}, \ldots, \boldsymbol{x}_{s}$. Moreover, since $M_{11}$ is an irreducible component, its dynamics is known, by $\mathbf{H}$. Let $\overline{\boldsymbol{x}}_{1}$ the corresponding globally asymptotically stable equilibrium. Now, we use a result of large decomposition system to determine the dynamics of $\boldsymbol{x}_{2}$. The outcome of this system is given by the following theorem.

Theorem 2.1. Let $\mathcal{C}_{1}$ be the strongly connected graph corresponding to the irreducible component $M_{11}$ and $\boldsymbol{d}_{1} \geq 0_{\mathbb{R}^{q}}$. If the global dynamics of the system

$$
\dot{\boldsymbol{x}}_{2}=f_{2}\left(\boldsymbol{d}_{1}, \boldsymbol{x}_{2}\right)
$$

is known, then the dynamics of the system

$$
\left\{\begin{array}{l}
\dot{\boldsymbol{x}}_{1}=f_{1}\left(\boldsymbol{x}_{1}\right) \\
\dot{\boldsymbol{x}}_{2}=f_{2}\left(\boldsymbol{x}_{1}, \boldsymbol{x}_{2}\right)
\end{array}\right.
$$

is completely characterized.

Proof. Since $\mathcal{C}_{1}$ is a strongly connected graph, its corresponding matrix $M_{11}$ is irreducible. Hence, by using $\mathbf{H}$, the global dynamics of the sub system $\dot{\boldsymbol{x}}_{1}=f_{1}\left(\boldsymbol{x}_{1}\right)$, as a projection of System (2.1) on $M_{11}$, is known. Let $\boldsymbol{d}_{1}$ a corresponding equilibrium which is globally asymptotically stable (GAS), potentiality under some conditions, denoted by $\mathcal{H}_{1}$. Now recall that the trajectories of (2.3) are bounded and that any equilibrium of $\dot{\boldsymbol{x}}_{2}=f_{2}\left(\boldsymbol{d}_{1}, \boldsymbol{x}_{2}\right)$ is globally asymptotically stable for any $\boldsymbol{d}_{1} \geq 0_{\mathbb{R}^{q}}$ (potentiality under some conditions, denoted by $\mathcal{H}_{2}$ ). By using a result of Vidyasagar [35] (Theorems 3.1 and 3.4), we conclude that the equilibrium $\left(\boldsymbol{d}_{1}, \boldsymbol{d}_{2}\right)$ is GAS for System (2.3), where $\boldsymbol{d}_{2}$ is the equilibrium of $\dot{\boldsymbol{x}}_{2}=f_{2}\left(\boldsymbol{d}_{1}, \boldsymbol{x}_{2}\right)$ that is GAS. This ends the proof.

By successively applying Theorem 2.1 on System (2.2), a complete characterization of the equilibria of System (2.2) will be obtained.

The most challenging part in applying Theorem 2.1 and thus characterize System (2.1), regardless of the network configuration is the study of the global stability of equilibria for the auxiliary system

$$
\dot{\boldsymbol{x}}_{j}=f_{j}\left(\boldsymbol{d}_{1}, \boldsymbol{d}_{2}, \ldots, \boldsymbol{d}_{j-1}, \boldsymbol{x}_{j}\right) .
$$

In the following section, we apply this method to two special models - namely a multi-host and multi-vector model (Sect. 3.1) and a patchy vector-borne model (Sect. 3.2). In these two cases, we assume that the network is weakly connected and investigate the global dynamics of their equilibria using the method described in Theorem 2.1.

It is worthwhile to note that earlier, we assumed WLOG that, for any $i, M_{i j} \neq 0$ for some $j=2, \ldots, i-1$. If $M_{i j}=0$ for some $j=2, \ldots, i-1$, then we define maximal and minimal irreducible components of the graph. Indeed, if the network is weakly connected, it is always possible to shrink the graph into a condensed graph with meta-nodes (see Appendix A). The edges from one meta-node to another describe the edges between the 
irreducible components. The matrices $M_{i j}$ for $i \neq j$ describes the edges between the meta-nodes. An irreducible component $\mathcal{C}_{i}$ is said to be minimal (or source) if it may influence the dynamics of other $\mathcal{C}_{j}$, with $j \neq i$, but not itself influenced by these $\mathcal{C}_{j}$, with $j \neq i$ (see Appendix A). In other words, an irreducible component is said to be minimal if it has no incoming edges. Otherwise, the irreducible component is called maximal. For instance, in Model 2.2, only $\mathcal{C}_{1}$ (or $M_{11}$ ) is minimal, since $\boldsymbol{x}_{1}$ influences the dynamics of $\boldsymbol{x}_{2}, \boldsymbol{x}_{3}, \ldots, \boldsymbol{x}_{s}$, but these do not influence the dynamics of $\boldsymbol{x}_{1}$. They are called maximal irreducible components. The case where there may be more than one minimal or maximal irreducible components is treated in the following section.

\section{Applications}

In this section, we apply the general method to two specific cases, namely on zoonoses model and vector-borne diseases in a patchy environment. These class of models involves the interactions of multiple subpopulations and their global dynamics are known when their connectivity matrices are irreducible. In the following two subsections, we successively determine the global dynamics when the network configurations are not irreducible. Particularly, we provide the complete global dynamics of the corresponding auxiliary systems that leads to the characterization of the general systems.

\subsection{The multi-host and multi-vector model}

Multi-vector zoonoses are diseases whose pathogen is shared by many host species and transmitted by one or multiple arthropods. Hence, capturing the dynamics of these types of diseases must account for the dynamics of all host and vector species involved, along with the infection processes. For generality's sake, we assume that the zoonoses' epidemiology follows an SEIR - SI structure. Moreover, we consider $m$ host species and $p$ arthropod species. The infection process is captured as follows. Susceptible hosts of species $i(1 \leq i \leq m)$, denoted by $S_{i}$, are infected by infectious arthropod of species $j(1 \leq j \leq p)$ through biting or landing, at a rate of $a_{i j}$ and an infectiousness of $\beta_{i, j}^{\diamond}$. Thus the host species $i$ 's infection rate is $\sum_{j=1}^{p} a_{i, j} \beta_{i, j}^{\diamond} S_{i} \frac{I_{v, j}}{N_{i}}$, where $N_{i}$ is the total population of host of species $i$. The susceptible arthropod vectors of species $j$, denoted by $S_{v, j}$, are infected by infectious host of species $i$. Hence, the rate of infection of $S_{v, j}$ is $\sum_{i=1}^{m} a_{i, j} \frac{\beta_{i, j} S_{v, j} I_{i}}{N_{i}}$. Susceptible hosts of species $i$ and vectors of species $j$ are recruited at a rate $\Lambda_{i}$ and $\Lambda_{v, j}$, respectively. Taken together, these assumptions lead to the system

$$
\left\{\begin{array}{l}
\dot{\boldsymbol{S}}=\boldsymbol{\Lambda}_{h}-\operatorname{diag}^{-1}\left(\boldsymbol{N}_{h}\right) \operatorname{diag}(\boldsymbol{S}) A \circ B^{\diamond} \boldsymbol{I}_{v}-\operatorname{diag}(\mu) \boldsymbol{S} \\
\dot{\boldsymbol{E}}=\operatorname{diag}^{-1}\left(\boldsymbol{N}_{h}\right) \operatorname{diag}(\boldsymbol{S}) A \circ B^{\diamond} \boldsymbol{I}_{v}-\operatorname{diag}(\mu+\nu) \boldsymbol{E} \\
\dot{\boldsymbol{I}}=\operatorname{diag}(\nu) \boldsymbol{E}-\operatorname{diag}(\gamma+\mu) \boldsymbol{I} \\
\dot{\boldsymbol{R}}=\operatorname{diag}(\gamma) \boldsymbol{I}-\operatorname{diag}(\mu) \boldsymbol{R} \\
\dot{\boldsymbol{S}}_{v}=\boldsymbol{\Lambda}_{v}-\operatorname{diag}\left(\boldsymbol{S}_{v}\right)(A \circ B)^{T} \operatorname{diag}^{-1}\left(\boldsymbol{N}_{h}\right) \boldsymbol{I}-\operatorname{diag}\left(\mu_{v}+\delta_{v}\right) \boldsymbol{S}_{v} \\
\dot{\boldsymbol{I}}_{v}=\operatorname{diag}\left(\boldsymbol{S}_{v}\right)(A \circ B)^{T} \operatorname{diag}^{-1}\left(\boldsymbol{N}_{h}\right) \boldsymbol{I}-\operatorname{diag}\left(\mu_{v}+\delta_{v}\right) \boldsymbol{I}_{v}
\end{array}\right.
$$

where $\circ$ denotes the Hadamard product, $\boldsymbol{S}=\left[S_{1}, S_{2}, \ldots, S_{m}\right]^{T}, \boldsymbol{E}=\left[E_{1}, E_{2}, \ldots, E_{m}\right]^{T}$ are the vectors of susceptible and latent host of all species and $\boldsymbol{I}=\left[I_{1}, I_{2}, \ldots, I_{m}\right]^{T}$ is the vector of infectious for all host species. For arthropod vectors, $\boldsymbol{S}_{v}=\left[S_{v, 1}, S_{v, 2}, \ldots, S_{v, p}\right]^{T}$ and $\boldsymbol{I}_{v}=\left[I_{v, 1}, I_{v, 2}, \ldots, I_{v, p}\right]^{T}$ denote the vectors of susceptible and infected respectively. To ease the notations, we denote $\alpha=\gamma+\mu$. The matrices $A, B^{\diamond}$ and $B$ are given by 
TABLE 1. Description of the parameters used in System (3.1).

\begin{tabular}{ll}
\hline Parameters & Description \\
\hline $\boldsymbol{\Lambda}_{\boldsymbol{h}}=\left[\Lambda_{1}, \Lambda_{2}, \ldots, \Lambda_{m}\right]^{T}$ & Vector of recruitment of the hosts; \\
$\boldsymbol{\Lambda}_{\boldsymbol{v}}=\left[\Lambda_{v, 1}, \Lambda_{v, 2}, \ldots, \Lambda_{v, p}\right]^{T}$ & Vector of recruitment of the vectors; \\
$a_{i, j}$ & Biting rate of vector $j$ on Host $i ;$ \\
$\beta_{i, j}^{\diamond}$ & Vector of species $j$ 's infectiousness to Host $i$ per biting; \\
$\mu=\left[\mu_{1}, \mu_{2}, \ldots, \mu_{m}\right]^{T}$ & Hosts' death rate; \\
$\nu=\left[\nu_{1}, \nu_{2}, \ldots, \nu_{m}\right]^{T}$ & Hosts' incubation rate; \\
$\alpha=\left[\alpha_{1}, \alpha_{2}, \ldots, \alpha_{m}\right]^{T}$ & Hosts' total infectiosity duration; \\
$\gamma=\left[\gamma_{1}, \gamma_{2}, \ldots, \gamma_{m}\right]^{T}$ & Hosts' recovery rate; \\
$\beta_{i, j}$ & Infectiousness of Host $i$ to vectors of species $j$ per biting; \\
$\mu_{v}=\left[\mu_{v, 1}, \mu_{v, 2}, \ldots, \mu_{v, p}\right]^{T}$ & Vectors' natural mortality rates; \\
$\delta_{v}=\left[\delta_{v, 1}, \delta_{v, 2}, \ldots, \delta_{v, p}\right]^{T}$ & Vectors' control-induced mortality rates. \\
\hline
\end{tabular}

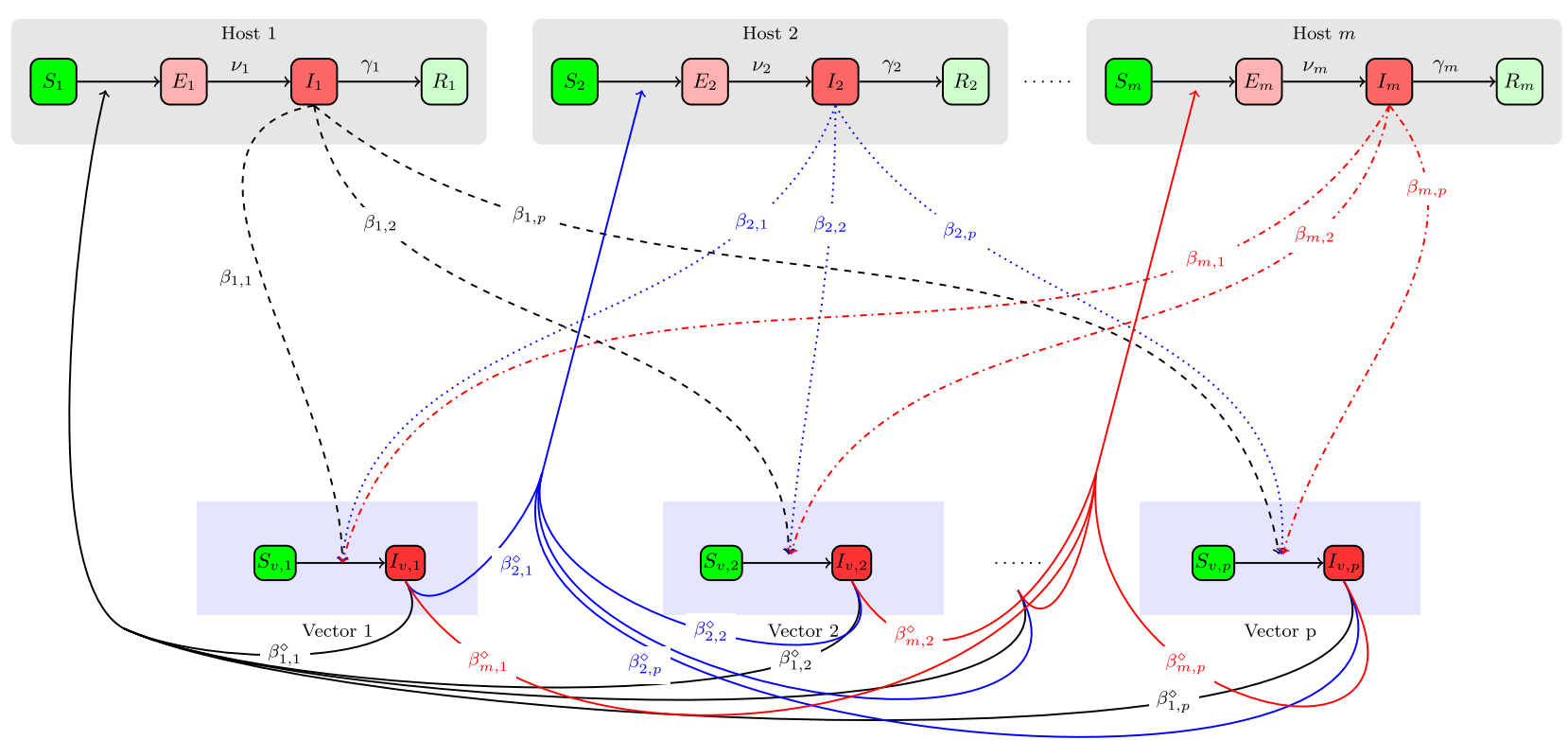

Figure 1. Flow diagram of Model 3.1. The dashed, dotted and dash-dotted lines capture the infection routes of vectors from all the infectious hosts. The solid lines capture the infection routes of hosts from the vectors.

$$
A=\left(\begin{array}{cccc}
a_{1,1} & a_{1,2} & \ldots & a_{1, p} \\
a_{2,1} & a_{2,2} & \ldots & a_{2, p} \\
\vdots & \vdots & \ddots & \vdots \\
a_{m, 1} & a_{m, 2} & \ldots & a_{m, p}
\end{array}\right), B^{\diamond}=\left(\begin{array}{cccc}
\beta_{1,1}^{\diamond} & \beta_{1,2}^{\diamond} & \ldots & \beta_{1, p}^{\diamond} \\
\beta_{2,1}^{\diamond} & \beta_{2,2}^{\diamond} & \ldots & \beta_{2, p}^{\diamond} \\
\vdots & \vdots & \ddots & \vdots \\
\beta_{m, 1}^{\diamond} & \beta_{m, 2}^{\diamond} & \ldots & \beta_{m, p}^{\diamond}
\end{array}\right), B=\left(\begin{array}{cccc}
\beta_{1,1} & \beta_{1,2} & \ldots & \beta_{1, p} \\
\beta_{2,1} & \beta_{2,2} & \ldots & \beta_{2, p} \\
\vdots & \vdots & \ddots & \vdots \\
\beta_{m, 1} & \beta_{m, 2} & \ldots & \beta_{m, p}
\end{array}\right)
$$

These matrices capture the biting rate, vector-to-host transmission, and host-to-vector transmission, respectively. The description of parameter vectors are summarized in Table 1, and a schematic description of flow of the model is captured by Figure 1. 
System (3.1) is the model considered in [8], for a single stage of infection. The dynamics of the total host and vector populations are given by $\dot{\boldsymbol{N}}_{h}=\boldsymbol{\Lambda}_{h}-\mu \boldsymbol{N}_{h}$ and $\dot{\boldsymbol{N}}_{v}=\boldsymbol{\Lambda}_{v}-\left(\mu_{v}+\delta_{v}\right) \boldsymbol{N}_{v}$. And so, these populations are asymptotically constant as $\boldsymbol{N}_{h} \rightarrow \frac{\boldsymbol{\Lambda}_{h}}{\mu}:=\overline{\boldsymbol{N}}_{h}$ and $\boldsymbol{N}_{v} \rightarrow \frac{\boldsymbol{\Lambda}_{v}}{\mu_{v}+\delta_{v}}:=\overline{\boldsymbol{N}}_{v}$ when $t \rightarrow \infty$. By using the theory of triangular systems, System (3.1) is equivalent to System (3.1) when $\boldsymbol{N}_{h}=\overline{\boldsymbol{N}}_{h}$ and $\boldsymbol{N}_{v}=\overline{\boldsymbol{N}}_{v}$.

The set

$$
\Omega=\left\{\left(\boldsymbol{S}, \boldsymbol{E}, \boldsymbol{I}, \boldsymbol{S}_{v}, \boldsymbol{I}_{v}\right) \in \mathbb{R}_{+}^{3 m+2 p} \mid \boldsymbol{S}+\boldsymbol{E}+\boldsymbol{I} \leq \boldsymbol{\Lambda}_{\boldsymbol{h}} \circ \frac{1}{\mu}, \boldsymbol{S}_{v}+\boldsymbol{I}_{v} \leq \boldsymbol{\Lambda}_{\boldsymbol{v}} \circ \frac{1}{\mu_{v}+\delta_{v}}\right\}
$$

is a compact attracting positively invariant set for System (3.1), where o denotes the Hadamard product. Therefore, the solutions of System (3.1) are biologically substantiated. The trivial equilibrium of System (3.1) is the disease-free equilibrium (D.F.E) and is given by $E_{0}=\left(\overline{\boldsymbol{S}}, \mathbf{0}_{2 m}, \overline{\boldsymbol{S}}_{v}, \mathbf{0}_{p}\right)$ where

$$
\overline{\boldsymbol{S}}=\boldsymbol{\Lambda}_{\boldsymbol{h}} \circ \frac{1}{\mu} \quad \text { and } \quad \overline{\boldsymbol{S}}_{v}=\boldsymbol{\Lambda}_{\boldsymbol{v}} \circ \frac{1}{\mu_{v}+\delta_{v}} .
$$

The basic reproduction number $\mathcal{R}_{0}$, defined as the average number of secondary cases produced by an infected individual during its infectious period while interacting with a purely susceptible population, can be computed using the next generation method [15,33]. The basic reproduction number of System (3.1) with $m$ hosts and $p$ vectors is given by:

$$
\begin{aligned}
\mathcal{R}_{0}^{2}(m, p) & =\rho\left(\mathcal{N}^{v}\right) \\
& =\rho\left(\mathcal{N}^{h}\right)
\end{aligned}
$$

where

$$
\mathcal{N}^{v}=\operatorname{diag}\left(\overline{\boldsymbol{N}}_{v}\right)\left((A \circ B)^{T} \operatorname{diag}(\nu) \operatorname{diag}^{-1}((\mu+\nu) \circ \alpha)\right) \operatorname{diag}^{-1}\left(\overline{\boldsymbol{N}}_{h}\right) A \circ B^{\diamond} \operatorname{diag}^{-1}\left(\mu_{v}+\delta_{v}\right)
$$

and

$$
\mathcal{N}^{h}=A \circ B^{\diamond} \operatorname{diag}^{-1}\left(\mu_{v}+\delta_{v}\right) \operatorname{diag}\left(\overline{\mathbf{N}}_{v}\right)(A \circ B)^{T} \operatorname{diag}(\nu) \operatorname{diag}^{-1}((\mu+\nu) \circ \alpha) \operatorname{diag}^{-1}\left(\overline{\mathbf{N}}_{h}\right)
$$

Indeed, by decomposing the infected compartments of (3.1) as a sum of new infection terms and transition terms,

$$
\begin{aligned}
\left(\begin{array}{c}
\dot{\boldsymbol{E}}_{\dot{\boldsymbol{I}}} \\
\dot{\boldsymbol{I}}_{v}
\end{array}\right) & =\mathcal{F}\left(\boldsymbol{E}, \boldsymbol{I}, \boldsymbol{I}_{v}\right)+\mathcal{V}\left(\boldsymbol{E}, \boldsymbol{I}, \boldsymbol{I}_{v}\right) \\
& =\left(\begin{array}{c}
\operatorname{diag}^{-1}\left(\overline{\boldsymbol{N}}_{h}\right) \operatorname{diag}(\boldsymbol{S}) A \circ B^{\diamond} \boldsymbol{I}_{v} \\
0 \\
\operatorname{diag}\left(\boldsymbol{S}_{v}\right)(A \circ B)^{T} \operatorname{diag}^{-1}\left(\overline{\boldsymbol{N}}_{h}\right) \boldsymbol{I}
\end{array}\right)+\left(\begin{array}{c}
-\operatorname{diag}(\mu+\nu) \boldsymbol{E} \\
\operatorname{diag}(\nu) \boldsymbol{E}-\operatorname{diag}(\alpha) \boldsymbol{I} \\
-\operatorname{diag}\left(\mu_{v}+\delta_{v}\right) \boldsymbol{I}_{v}
\end{array}\right)
\end{aligned}
$$

The Jacobian matrices at the D.F.E $\left(E_{0}\right)$ of $\mathcal{F}\left(\boldsymbol{E}, \boldsymbol{I}, \boldsymbol{I}_{v}\right)$ and $\mathcal{V}\left(\boldsymbol{E}, \boldsymbol{I}, \boldsymbol{I}_{v}\right)$ are given by:

$$
F=\left.D \mathcal{F}\left(\boldsymbol{E}, \boldsymbol{I}, \boldsymbol{I}_{v}\right)\right|_{E_{0}}=\left(\begin{array}{ccc}
\mathbf{0}_{m, m} & \mathbf{0}_{m, m} & A \circ B^{\diamond} \\
\mathbf{0}_{m, m} & \mathbf{0}_{m, m} & \mathbf{0}_{m, p} \\
\mathbf{0}_{p, m} & \operatorname{diag}\left(\overline{\boldsymbol{N}}_{v}\right)(A \circ B)^{T} \operatorname{diag}^{-1}\left(\overline{\mathbf{N}}_{h}\right) & \mathbf{0}_{p, p}
\end{array}\right)
$$


and,

$$
V=\left.D \mathcal{V}\left(\boldsymbol{E}, \boldsymbol{I}, \boldsymbol{I}_{v}\right)\right|_{E_{0}}=\left(\begin{array}{ccc}
-\operatorname{diag}(\mu+\nu) & \mathbf{0}_{m, m} & \mathbf{0}_{m, p} \\
\operatorname{diag}(\nu) & -\operatorname{diag}(\alpha) & \mathbf{0}_{m, p} \\
\mathbf{0}_{p, m} & \mathbf{0}_{p, m} & -\operatorname{diag}\left(\mu_{v}+\delta_{v}\right)
\end{array}\right)
$$

Hence, we obtain

$$
-V^{-1}=\left(\begin{array}{ccc}
\operatorname{diag}^{-1}(\mu+\nu) & \mathbf{0}_{m, m} & \mathbf{0}_{m, p} \\
\operatorname{diag}(\nu) \operatorname{diag}^{-1}((\mu+\nu) \circ \alpha) & \operatorname{diag}^{-1}(\alpha) & \mathbf{0}_{m, p} \\
\mathbf{0}_{p, m} & \mathbf{0}_{p, m} & \operatorname{diag}^{-1}\left(\mu_{v}+\delta_{v}\right)
\end{array}\right)
$$

The basic reproduction number is the spectral radius of the next generation matrix

$$
-F V^{-1}=\left(\begin{array}{ccc}
\mathbf{0}_{m, m} & \mathbf{0}_{m, m} & Z_{v h} \operatorname{diag}^{-1}\left(\mu_{v}+\delta_{v}\right) \\
\mathbf{0}_{m, m} & \mathbf{0}_{m, m} & \mathbf{0}_{m, p} \\
Z_{h v} \operatorname{diag}(\nu) \operatorname{diag}^{-1}((\mu+\nu) \circ \alpha) & Z_{h v} \operatorname{diag}(\alpha) & \mathbf{0}_{p, p}
\end{array}\right),
$$

where $Z_{h v}=\operatorname{diag}\left(\overline{\boldsymbol{N}}_{v}\right)(A \circ B)^{T} \operatorname{diag}^{-1}\left(\overline{\boldsymbol{N}}_{h}\right)$ and $Z_{v h}=A \circ B^{\diamond}$. Thus, the basic reproduction number is

$$
\begin{aligned}
\mathcal{R}_{0} & =\rho\left(Z_{h v} \operatorname{diag}(\nu) \operatorname{diag}^{-1}((\mu+\nu) \circ \alpha) Z_{v h} \operatorname{diag}^{-1}\left(\mu_{v}+\delta_{v}\right)\right) \\
& =\rho\left(Z_{v h} \operatorname{diag}^{-1}\left(\mu_{v}+\delta_{v}\right) Z_{h v} \operatorname{diag}(\nu) \operatorname{diag}^{-1}((\mu+\nu) \circ \alpha)\right) .
\end{aligned}
$$

The dynamics of the multi-host and multi-vector is tied on how the different units of the model are interconnected. That is, the connectivity between host species and vector species. For Model (3.1), the host-vector configuration network is given by $\mathcal{N}$, where:

$$
\mathcal{N}=\left(\begin{array}{cc}
\mathbf{0}_{2 m, 2 m} & A \circ B^{\diamond} \\
(A \circ B)^{T} & \mathbf{0}_{m+p, p}
\end{array}\right) .
$$

Indeed, it has been shown in [8] that, for System (3.1), the disease either dies out or persists in all hosts and vectors, under the assumption that the Host-Vector network configuration is strongly connected. For the convenience of the reader, we recall the following theorem that summarizes the results in [8].

Theorem 3.1 ([8]). If the Host-Vector connectivity configuration $\mathcal{N}$ is irreducible, the following hold:

1. If $\mathcal{R}_{0}^{2}(m, p) \leq 1$, the DFE is $G A S$.

2. If $\mathcal{R}_{0}^{2}(m, p)>1$, a unique interior endemic equilibrium exists and is GAS.

The connectivity matrix $\mathcal{N}$ is irreducible if, and only if $(A \circ B)^{T} A \circ B^{\diamond}$ and $A \circ B^{\diamond}(A \circ B)^{T}$ are irreducible. However, for zoonoses this irreducibility assumption is not always satisfied as many host species and vector species, though share the same pathogen, have their natural habitats far away from each others, for a direct infection to take place. Therefore, it is important to investigate the dynamics of the disease when the network configuration between hosts and vector species is not strongly connected. 


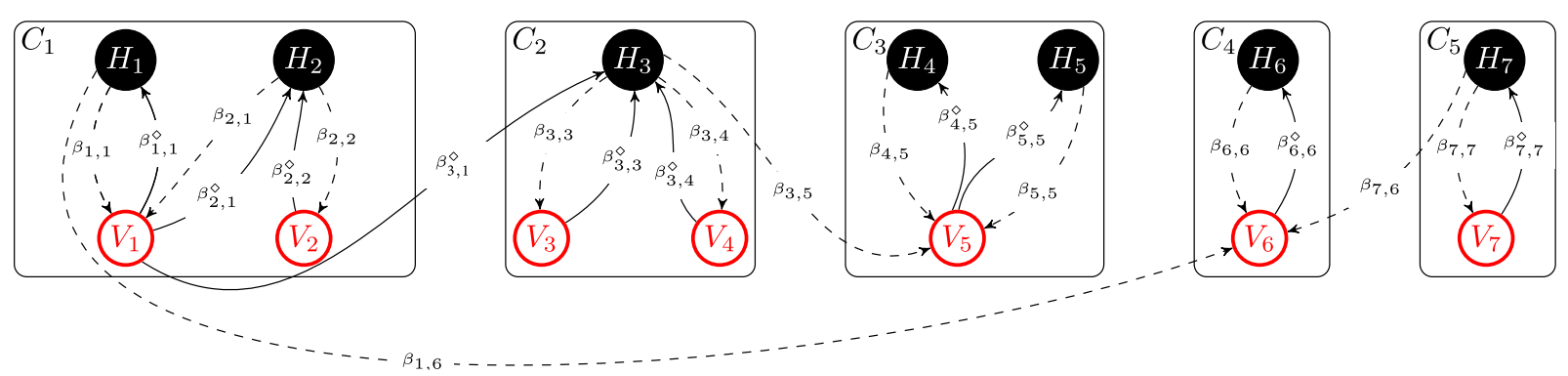

Figure 2. Digraph of $V(\mathcal{C})$. This is the same as Figure 1, with $n=p=7$, and the missing arrows between host and vector species means the infectiousness rates between them is set to be zero. The graph is re-arranged in terms of connected components $\mathcal{C}_{i}$.

Throughout the rest of the paper, we consider System (3.1) in its component-wise form. That is, for $i=$ $1, \ldots, m$ and $j=1, \ldots, p$,

$$
\left\{\begin{array}{l}
\dot{S}_{i}=\Lambda_{i}-\sum_{j=1}^{p} a_{i, j} \beta_{i, j}^{\diamond} S_{i} \frac{I_{v, j}}{N_{i}}-\mu_{i} S_{i} \\
\dot{E}_{i}=\sum_{j=1}^{p} a_{i, j} \beta_{i, j}^{\diamond} S_{i} \frac{I_{v, j}}{N_{i}}-\left(\mu_{i}+\nu_{i}\right) E_{i} \\
\dot{I}_{i}=\nu_{i} E_{i}-\alpha_{i} I_{i} \\
\dot{S}_{v, j}=\Lambda_{v, j}-\sum_{i=1}^{m} a_{i, j} \beta_{i, j} S_{v, j} \frac{I_{i}}{N_{i}}-\left(\mu_{v, j}+\delta_{v, j}\right) S_{v, j} \\
\dot{I}_{v, j}=\sum_{i=1}^{m} a_{i, j} \beta_{i, j} S_{v, j} \frac{I_{i}}{N_{i}}-\left(\mu_{v, j}+\delta_{v, j}\right) I_{v, j}
\end{array}\right.
$$

The following theorem gives the main result of the paper, which characterizes the dynamics of System (3.2), regardless of the network configuration.

\section{Theorem 3.2.}

1. If $\mathcal{R}_{0}^{2}(m, p) \leq 1$, the DFE is $G A S$.

2. If $\mathcal{R}_{0}^{2}(m, p)>1$, a unique endemic equilibrium, potentially mixed or interior, is GAS.

Theorem 3.2 provides a global result of equilibria of System (3.2). Particularly, Theorem 3.2, Item 2 arises the possible existence of boundary equilibria and determines their global behavior. This result is obtained without any hypothesis on the structure of the host-vector network configuration and thus generalizes the result in [8] where host-vector configuration is irreducible.

In our model and with vector-borne diseases in general, an infection takes place if at least there is an interaction between a vector and host. So, we define a node as unit with at least one host and one vector.

With the relaxation of the irreducibility hypothesis in mind, let us re-organize the host-vector network in a way that makes it as a set of strongly connected component (see Fig. 2). Suppose that there are $l$ strongly connected components $\mathcal{C}_{i}$, for $i=1,2, \ldots, l$. If $l=1$, the host-vector network is irreducible, which is dealt with in [8] and the dynamics of the model is completely captured by Theorem 3.1. We assume that $l \geq 2$. Let $V(\mathcal{C})=\left\{\mathcal{C}_{i}, i=1,2, \ldots, l\right\}$, the set of all $\mathcal{C}_{i}$. It could be shown that the basic reproduction number for System (3.1), is:

$$
\mathcal{R}_{0}^{2}(m, p)=\max _{1 \leq i \leq l}\left\{\mathcal{R}_{0, \mathcal{C}_{i}}^{2}, \quad \mathcal{C}_{i} \in V(\mathcal{C})\right\}
$$




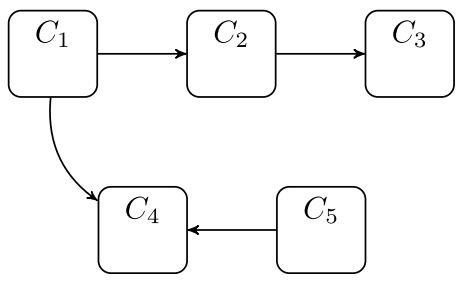

Figure 3. Directed graph of the condensed network $V(\mathcal{C})$.

- An element $\mathcal{C}_{i}$ of $V(\mathcal{C})$ is called minimal if it may influence the dynamics of other $\mathcal{C}_{j}$, with $j \neq i$, but not itself influenced by these $\mathcal{C}_{j}$, with $j \neq i$. For instance, in Figure $2, \mathcal{C}_{1}$ and $\mathcal{C}_{5}$ are minimal. The elements $\mathcal{C}_{j}$, with $j \neq 1$ and $j \neq 5$ are called maximal.

The following theorem provides a building-block to prove Theorem 3.2.

Theorem 3.3. Let $\mathcal{C}$ be a strongly connected component with $m^{\prime}$ hosts and $p^{\prime}$ vectors, $m^{\prime} \leq m$ and $p^{\prime} \leq p$. Then, for any vector $(\tilde{d}, d)^{T} \geq 0$, the System

$$
\left\{\begin{array}{l}
\dot{S}_{i}=\Lambda_{i}-\sum_{j=1}^{p^{\prime}} a_{i, j} \beta_{i, j}^{\diamond} S_{i} \frac{I_{v, j}}{N_{i}}-\sum_{j=p^{\prime}+1}^{p} a_{i, j} \beta_{i, j}^{\diamond} S_{i} \frac{d_{j}}{N_{i}}-\mu_{i} S_{i} \\
\dot{E}_{i}=\sum_{j=1}^{p^{\prime}} a_{i, j} \beta_{i, j}^{\diamond} S_{i} \frac{I_{v, j}}{N_{i}}+\sum_{j=p^{\prime}+1}^{p} a_{i, j} \beta_{i, j}^{\diamond} S_{i} \frac{d_{j}}{N_{i}}-\left(\mu_{i}+\nu_{i}\right) E_{i} \\
\dot{I}_{i}=\nu_{i} E_{i}-\alpha_{i} I_{i} \\
\dot{S}_{v, j}=\Lambda_{v, j}-\sum_{i=1}^{m^{\prime}} a_{i, j} \beta_{i, j} S_{v, j} \frac{I_{i}}{N_{i}}-\sum_{i=m^{\prime}+1}^{m} a_{i, j} \beta_{i, j} S_{v, j} \frac{\tilde{d}_{i}}{N_{i}}-\left(\mu_{v, j}+\delta_{v, j}\right) S_{v, j} \\
\dot{I}_{v, j}=\sum_{i=1}^{m^{\prime}} a_{i, j} \beta_{i, j} S_{v, j} \frac{I_{i}}{N_{i}}+\sum_{i=m^{\prime}+1}^{m} a_{i, j} \beta_{i, j} S_{v, j} \frac{\tilde{d}_{i}}{N_{i}}-\left(\mu_{v, j}+\delta_{v, j}\right) I_{v, j}
\end{array}\right.
$$

has the the following behavior

- If $\left.\mathcal{R}_{0}^{2}\left(m^{\prime}, p^{\prime}\right)\right|_{(3.3)}>1$ or $(\tilde{d}, d)^{T}>0$, there is a unique interior endemic equilibrium that is GAS.

- If $\left.\mathcal{R}_{0}^{2}\left(m^{\prime}, p^{\prime}\right)\right|_{(3.3)} \leq 1$ and $(\tilde{d}, d)^{T}=0$, then the DFE is GAS.

System (3.3) is called the restricted system on $\mathcal{C}$ at $(\tilde{d}, d)^{T}$.

Remark 3.4. By denoting $\left.\mathcal{R}_{0}^{2}\left(m^{\prime}, p^{\prime}\right)\right|_{(3.3)}$ the basic reproduction number of (3.3), it is straightforward to show that:

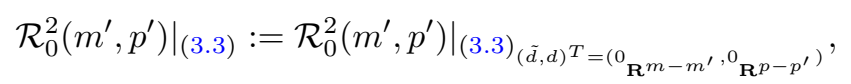

where $(3.3)_{(\tilde{d}, d)^{T}=\left(0_{\mathbf{R}^{m-m^{\prime}}, 0} \mathbf{R}^{p-p^{\prime}}\right)}$ is System (3.3) evaluated at $(\tilde{d}, d)^{T}=\left(0_{\mathbf{R}^{m-m^{\prime}}}, 0_{\mathbf{R}^{p-p^{\prime}}}\right)$.

The following result links the dynamics of System (3.3) to that of System (3.2).

Theorem 3.5. The asymptotic behavior of System (3.3) is isomorphic to that of System (3.2).

Proof. We re-arrange $V(\mathcal{C})$ so that there are $k$ minimal elements $\mathcal{C}_{1}, \mathcal{C}_{2}, \ldots, \mathcal{C}_{k}$ and $r-k$ maximal elements $\mathcal{C}_{k+1}, \mathcal{C}_{k+2}, \ldots, \mathcal{C}_{r}$, with $r=|V(\mathcal{C})|$.

Let $\boldsymbol{x}_{q}=\left(S_{1}, S_{2} \ldots, S_{i(q)}, E_{1}, E_{2} \ldots, E_{i(q)}, I_{1}, I_{2} \ldots, I_{i(q)}, S_{v, 1}, S_{v, 2} \ldots, S_{v, j(q)}, I_{v, 1}, I_{v, 2} \ldots, I_{v, j(q)}\right)^{T} \in \mathcal{C}_{q}$. 
Here $i(q)$ and $j(q)$ are the number of hosts and vectors in $\mathcal{C}_{q}$, respectively. Therefore, given that $\mathcal{C}_{q}$, for $1 \leq q \leq k$, are minimal, System (3.2) could be written as:

$$
\left\{\begin{array}{l}
\dot{\boldsymbol{x}}_{1}=f_{1}\left(\boldsymbol{x}_{1}\right) \\
\dot{\boldsymbol{x}}_{2}=f_{2}\left(\boldsymbol{x}_{2}\right) \\
\vdots \\
\dot{\boldsymbol{x}}_{k}=f_{k}\left(\boldsymbol{x}_{k}\right) \\
\\
\dot{\boldsymbol{x}}_{k+1}=f_{k+1}\left(\boldsymbol{x}_{1}, \ldots, \boldsymbol{x}_{k}, \boldsymbol{x}_{k+1}, \ldots, \boldsymbol{x}_{r}\right) \\
\vdots \\
\dot{\boldsymbol{x}}_{r}=f_{r}\left(\boldsymbol{x}_{1}, \ldots, \boldsymbol{x}_{k}, \boldsymbol{x}_{k+1}, \ldots, \boldsymbol{x}_{r}\right)
\end{array}\right.
$$

The first $k$ subsystems of System (3.4) are independent of each others's variables and the variables of the remaining $r-k$ subsystems. Moreover, for each $q \in\{1, \ldots, k\}, \mathcal{C}_{q}$ is strongly connected and thus the corresponding host-vector configuration is irreducible. Therefore, the dynamics of the subsystems $\dot{\boldsymbol{x}}_{q}=f_{q}\left(\boldsymbol{x}_{q}\right)$, for $q \in\{1, \ldots, k\}$, are known (see Thm. 3.2) as follows:

- If $\mathcal{R}_{0, \mathcal{C}_{q}}^{2} \leq 1$, the corresponding disease-free equilibrium, $\mathrm{DFE}_{q}$, is GAS.

- If $\mathcal{R}_{0, \mathcal{C}_{q}}^{2}>1$, a unique positive endemic equilibrium $\bar{x}_{q}$ is GAS.

Denoting $\boldsymbol{x}_{q}^{*}\left(\boldsymbol{x}_{q}^{*}=\boldsymbol{x}_{q}^{0}\right.$ if the equilibrium is the $\mathrm{DFE}_{\mathrm{q}}$ or $\boldsymbol{x}_{q}^{*}=\overline{\boldsymbol{x}}_{q}$, if the equilibrium is endemic) the unique GAS equilibrium for $\dot{\boldsymbol{x}}_{q}=f_{q}\left(\boldsymbol{x}_{q}\right)$ (for $\left.1 \leq q \leq k\right)$, the asymptotic behavior of System (3.4) can be obtained, using Vidyasagar's decomposition techniques [35], by studying the system

$$
\left\{\begin{array}{l}
\dot{\boldsymbol{x}}_{k+1}=f_{k+1}\left(\boldsymbol{x}_{1}^{*}, \ldots, \boldsymbol{x}_{k}^{*}, \boldsymbol{x}_{k+1}, \ldots, \boldsymbol{x}_{r}\right) \\
\vdots \\
\dot{\boldsymbol{x}}_{r}=f_{r}\left(\boldsymbol{x}_{1}^{*}, \ldots, \boldsymbol{x}_{k}^{*}, \boldsymbol{x}_{k+1}, \ldots, \boldsymbol{x}_{r}\right)
\end{array}\right.
$$

Now, we consider System (3.5) as a new system, which is an autonomous system whose state variables are $\boldsymbol{x}_{k+1}, \ldots, \boldsymbol{x}_{r}$ (the $\boldsymbol{x}_{q}^{*}, \ldots, \boldsymbol{x}_{k}^{*}$ are constant). System (3.5) is associated with a new network configuration $V(\mathcal{C})$ -where the input connexions from the minimal elements of $V(\mathcal{C})$ have been cut.

If the new network configuration associated with System 3.5 is strongly connected, then it is equivalent to System (3.3). Otherwise, we repeat the process. For example, this case is illustrated in Figure 4, after the first iteration of the method on the network given in Figure 2. Indeed, in the network configuration of Figure 2, $\mathcal{C}_{1}$ and $\mathcal{C}_{5}$ are the minimal elements, and so after the first iteration, the network associated with the corresponding System 3.5 is illustrated in Figure 4.

We identify the minimal elements $\left(\mathcal{C}_{2}\right.$ in Fig. 4 is a minimal element and $\mathcal{C}_{4}$ is decoupled from the rest of the system) on the new network $V(\mathcal{C})$ and we reiterate the method. Repeating this process leads to reduce the study of the dynamics inside one irreducible component, say $s$. We obtain the system:

$$
\dot{\boldsymbol{x}}_{s}=f_{s}\left(\boldsymbol{x}_{1}^{*}, \ldots, \boldsymbol{x}_{k}^{*}, \boldsymbol{x}_{k+1}^{*}, \ldots, \boldsymbol{x}_{s-1}^{*}, \boldsymbol{x}_{s}, \boldsymbol{x}_{s+1}^{*}, \ldots, \boldsymbol{x}_{r}^{*}\right),
$$

which is System (3.3) with $\left(\tilde{d}_{i}, d_{i}\right)$ being the corresponding coordinates of $\boldsymbol{x}_{q}^{*}, q \neq s$. This ends the proof of Theorem 3.5.

Proof of Theorem 3.3. If $(\tilde{d}, d)^{T}=0$, the global asymptotic stability is known thanks to Hypothesis H. Particularly, if $\left.\mathcal{R}_{0}^{2}\left(m^{\prime}, p^{\prime}\right)\right|_{(3.3)} \leq 1$, the DFE is GAS. If $\left.\mathcal{R}_{0}^{2}\left(m^{\prime}, p^{\prime}\right)\right|_{(3.3)}>1$ and $(\tilde{d}, d)^{T}=0$, then it exists an interior equilibrium that is GAS, by Hypothesis $\mathbf{H}$. 

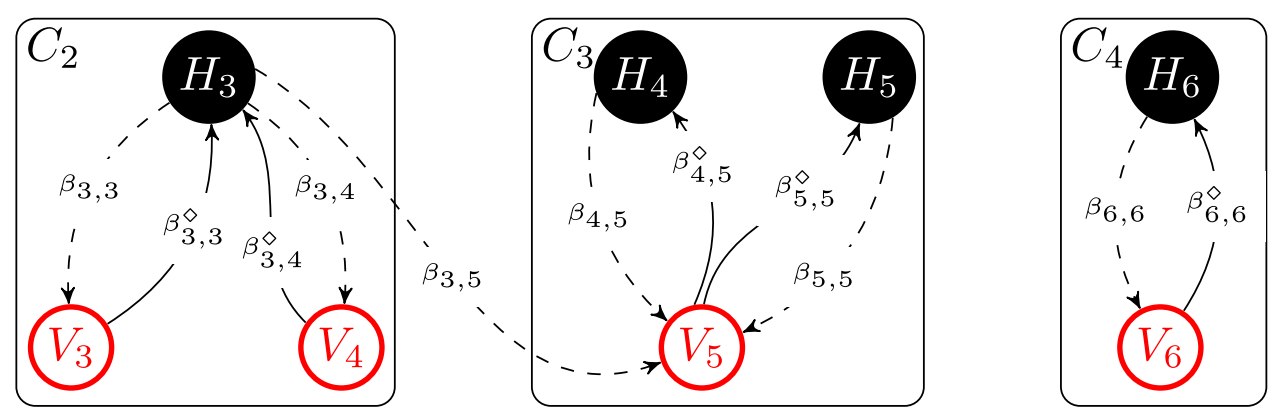

FIgURE 4. Network configuration after the first iteration of the reduction method on Figure 2.

The compact convex set $\Omega$ is positively invariant for System (3.3). Therefore System (3.3) has an equilibrium $\left(S^{*}, E^{*}, I^{*}, S_{v}^{*}, I_{v}^{*}\right)$ in $\Omega$ thanks to Brouwer fixed-point theorem. It is clear here that $S^{*} \gg 0$ and $S_{v}^{*} \gg 0$. For $i=1,2, \ldots, m^{\prime}$, we have:

$$
\frac{S_{i}^{*}}{N_{i}}\left(\sum_{j=1}^{p^{\prime}} a_{i, j} \beta_{i, j}^{\diamond} I_{v, j}^{*}+\sum_{j=p^{\prime}+1}^{p} a_{i, j} \beta_{i, j}^{\diamond} d_{j}\right)=\left(\mu_{i}+\nu_{i}\right) E_{i}^{*} .
$$

It then follows that $E_{i}^{*}>0$ if at least one $d_{j}$ is non-zero. Therefore $I_{i}^{*}=\frac{\nu_{i}}{\alpha_{i}} E_{i}^{*}>0$. The same reasoning shows that $I_{v}^{*} \gg 0$.

We consider the following candidate Lyapunov function:

$$
\mathcal{V}=\sum_{i=1}^{m} v_{i} \mathcal{V}_{i}
$$

where

$$
\mathcal{V}_{i}=\int_{S_{i}^{*}}^{S_{i}}\left(1-\frac{S_{i}^{*}}{x}\right) \mathrm{d} x+\int_{E_{i}^{*}}^{E_{i}}\left(1-\frac{E_{i}^{*}}{x}\right) \mathrm{d} x+\frac{\nu_{i}+\mu_{i}}{\nu_{i}} \int_{I_{i}^{*}}^{I_{i}}\left(1-\frac{I_{i}^{*}}{x}\right) \mathrm{d} x+\sum_{j=1}^{p} w_{i j} \mathcal{V}_{v, j}
$$

with

$$
\mathcal{V}_{v, j}=\int_{S_{v, j}^{*}}^{S_{v, j}}\left(1-\frac{S_{v, j}^{*}}{x}\right) \mathrm{d} x+\int_{I_{v, j}^{*}}^{I_{v, j}}\left(1-\frac{I_{v, j}^{*}}{x}\right) \mathrm{d} x, \quad w_{i j}=\frac{a_{i, j} \beta_{i, j}^{\diamond} S_{i}^{*} \frac{I_{v, j}^{*}}{N_{i}}}{\sum_{l=1}^{m^{\prime}} a_{l, j} \beta_{l, j} S_{v, j}^{*} \frac{I_{l}^{*}}{N_{l}}} .
$$

The derivative of the "Host" part of $\mathcal{V}_{i}$ is given by:

$$
\begin{aligned}
\dot{\mathcal{V}}_{h, i} & =\left(1-\frac{S_{i}^{*}}{S_{i}}\right) \dot{S}_{i}+\left(1-\frac{E_{i}^{*}}{E_{i}}\right) \dot{E}_{i}+\frac{\nu_{i}+\mu_{i}}{\nu_{i}}\left(1-\frac{I_{i}^{*}}{I_{i}}\right) \dot{I}_{i} \\
& =\Lambda_{i}-\mu_{i} S_{i}-\left(\mu_{i}+\nu_{i}\right) E_{i}-\frac{S_{i}^{*}}{S_{i}} \dot{S}_{i}-\frac{E_{i}^{*}}{E_{i}} \dot{E}_{i}+\frac{\nu_{i}+\mu_{i}}{\nu_{i}}\left(1-\frac{I_{i}^{*}}{I_{i}}\right) \dot{I}_{i}
\end{aligned}
$$


By using the endemic relation stemming from the equation of $S$, and some re-arrangement, equation (3.6) leads to:

$$
\begin{aligned}
\dot{\mathcal{V}}_{h, i}= & \mu_{i} S_{i}^{*}\left(2-\frac{S_{i}^{*}}{S_{i}}-\frac{S_{i}}{S_{i}^{*}}\right)+\sum_{j=1}^{p^{\prime}} a_{i, j} \beta_{i, j}^{\diamond} S_{i}^{*} \frac{I_{v, j}^{*}}{N_{i}}\left(2-\frac{S_{i}^{*}}{S_{i}}-\frac{S_{i}}{S_{i}^{*}} \frac{I_{v, j}}{I_{v, j}^{*}} \frac{E_{i}^{*}}{E_{i}}\right) \\
& +\sum_{j=p^{\prime}+1}^{p} a_{i, j} \beta_{i, j}^{\diamond} S_{i}^{*} \frac{d_{j}}{N_{i}}\left(3-\frac{S_{i}^{*}}{S_{i}}-\frac{S_{i}}{S_{i}^{*}} \frac{E_{i}^{*}}{E_{i}}\right)+\sum_{j=1}^{p^{\prime}} a_{i, j} \beta_{i, j}^{\diamond} S_{i}^{*} \frac{I_{v, j}}{N_{i}} \\
& -\left(\nu_{i}+\mu_{i}\right) E_{i}^{*} \frac{I_{i}}{I_{i}^{*}}-\left(\nu_{i}+\mu_{i}\right) E_{i}^{*} \frac{I_{i}^{*}}{I_{i}} \frac{E_{i}}{E_{i}^{*}}+\left(\nu_{i}+\mu_{i}\right) E_{i}^{*}
\end{aligned}
$$

Moreover, by using the endemic relationship that stem from the equation of $E$ :

$$
\left(\mu_{i}+\nu_{i}\right) E_{i}^{*}=\sum_{j=1}^{p^{\prime}} a_{i, j} \beta_{i, j}^{\diamond} S_{i}^{*} \frac{I_{v, j}^{*}}{N_{i}}+\sum_{j=p^{\prime}+1}^{p} a_{i, j} \beta_{i, j}^{\diamond} S_{i}^{*} \frac{d_{j}}{N_{i}},
$$

and some careful combinations, we obtain, from equation (3.7),

$$
\begin{aligned}
\dot{\mathcal{V}}_{h, i}= & \mu_{i} S_{i}^{*}\left(2-\frac{S_{i}^{*}}{S_{i}}-\frac{S_{i}}{S_{i}^{*}}\right)+\sum_{j=1}^{p^{\prime}} a_{i, j} \beta_{i, j}^{\diamond} S_{i}^{*} \frac{I_{v, j}^{*}}{N_{i}}\left(3-\frac{S_{i}^{*}}{S_{i}}-\frac{S_{i}}{S_{i}^{*}} \frac{I_{v, j}}{I_{v, j}^{*}} \frac{E_{i}^{*}}{E_{i}}-\frac{I_{i}^{*}}{I_{i}} \frac{E_{i}}{E_{i}^{*}}\right) \\
& +\sum_{j=p^{\prime}+1}^{p} a_{i, j} \beta_{i, j}^{\diamond} S_{i}^{*} \frac{d_{j}}{N_{i}}\left(4-\frac{S_{i}^{*}}{S_{i}}-\frac{S_{i}}{S_{i}^{*}} \frac{E_{i}^{*}}{E_{i}}-\frac{I_{i}^{*}}{I_{i}} \frac{E_{i}}{E_{i}^{*}}-\frac{I_{i}}{I_{i}^{*}}\right)+\sum_{j=1}^{p^{\prime}} a_{i, j} \beta_{i, j}^{\diamond} S_{i}^{*} \frac{I_{v, j}}{N_{i}} \\
& -\left[\sum_{j=1}^{p^{\prime}} a_{i, j} \beta_{i, j}^{\diamond} S_{i}^{*} \frac{I_{v, j}^{*}}{N_{i}}\right] \frac{I_{i}}{I_{i}^{*}} .
\end{aligned}
$$

Now, we will determine the derivative of vector part of $\mathcal{V}_{i}$. That is, $\mathcal{V}_{v, j}$ where

$$
\mathcal{V}_{v, j}=\int_{S_{v, j}^{*}}^{S_{v, j}}\left(1-\frac{S_{v, j}^{*}}{x}\right) \mathrm{d} x+\int_{I_{v, j}^{*}}^{I_{v, j}}\left(1-\frac{I_{v, j}^{*}}{x}\right) \mathrm{d} x .
$$

We obtain,

$$
\begin{aligned}
\dot{\mathcal{V}}_{v, j}= & \left(1-\frac{S_{v, j}^{*}}{S_{v, j}}\right) \dot{S}_{v, j}+\left(1-\frac{I_{v, j}^{*}}{I_{v, j}}\right) \dot{I}_{v, j}=\left(\dot{S}_{v, j}+\dot{I}_{v, j}-\frac{S_{v, j}^{*}}{S_{v, j}} \dot{S}_{v, j}-\frac{I_{v, j}^{*}}{I_{v, j}} \dot{I}_{v, j}\right) \\
= & \left(\mu_{v, j}+\delta_{v, j}\right) S_{v, j}^{*}\left(2-\frac{S_{v, j}^{*}}{S_{v, j}}-\frac{S_{v, j}}{S_{v, j}^{*}}\right)+\sum_{i=1}^{m^{\prime}} a_{i, j} \beta_{i, j} S_{v, j}^{*} \frac{I_{i}^{*}}{N_{i}}\left(1-\frac{S_{v, j}^{*}}{S_{v, j}}-\frac{I_{v, j}^{*}}{I_{v, j}} \frac{S_{v, j}}{S_{v, j}^{*}} \frac{I_{i}}{I_{i}^{*}}\right) \\
& +\sum_{i=m^{\prime}+1}^{m} a_{i, j} \beta_{i, j} S_{v, j}^{*} \frac{\tilde{d}_{i}}{N_{i}}\left(2-\frac{S_{v, j}^{*}}{S_{v, j}}-\frac{I_{v, j}^{*}}{I_{v, j}} \frac{S_{v, j}}{S_{v, j}^{*}}\right) \\
& -\left(\mu_{v, j}+\delta_{v, j}\right) I_{v, j}+\sum_{i=1}^{m^{\prime}} a_{i, j} \beta_{i, j} S_{v, j}^{*} \frac{I_{i}}{N_{i}}+\left(\mu_{v, j}+\delta_{v, j}\right) I_{v, j}^{*}
\end{aligned}
$$


However, we have $\left(\mu_{v, j}+\delta_{v, j}\right) I_{v, j}^{*}=\sum_{i=1}^{m^{\prime}} a_{i, j} \beta_{i, j} S_{v, j}^{*} \frac{I_{i}^{*}}{N_{i}}+\sum_{i=m^{\prime}+1}^{m} a_{i, j} \beta_{i, j} S_{v, j}^{*} \frac{\tilde{d}_{i}}{N_{i}}$.

Therefore, equation (3.9) gives:

$$
\begin{aligned}
\dot{\mathcal{V}}_{v, j}= & \left(\mu_{v, j}+\delta_{v, j}\right) S_{v, j}^{*}\left(2-\frac{S_{v, j}^{*}}{S_{v, j}}-\frac{S_{v, j}}{S_{v, j}^{*}}\right)+\sum_{i=1}^{m^{\prime}} a_{i, j} \beta_{i, j} S_{v, j}^{*} \frac{I_{i}^{*}}{N_{i}}\left(2-\frac{S_{v, j}^{*}}{S_{v, j}}-\frac{I_{v, j}^{*}}{I_{v, j}} \frac{S_{v, j}}{S_{v, j}^{*}} \frac{I_{i}}{I_{i}^{*}}\right) \\
& +\sum_{i=m^{\prime}+1}^{m} a_{i, j} \beta_{i, j} S_{v, j}^{*} \frac{\tilde{d}_{i}}{N_{i}}\left(3-\frac{S_{v, j}^{*}}{S_{v, j}}-\frac{I_{v, j}^{*}}{I_{v, j}} \frac{S_{v, j}}{S_{v, j}^{*}}-\frac{I_{v, j}}{I_{v, j}^{*}}\right)-\left(\sum_{i=1}^{m^{\prime}} a_{i, j} \beta_{i, j} S_{v, j}^{*} \frac{I_{i}^{*}}{N_{i}}\right) \frac{I_{v, j}}{I_{v, j}^{*}} \\
& +\sum_{i=1}^{m^{\prime}} a_{i, j} \beta_{i, j} S_{v, j}^{*} \frac{I_{i}}{N_{i}}
\end{aligned}
$$

Now, the overall derivative of the Lyapunov candidate is, using (3.8) and (3.10):

$$
\begin{aligned}
\dot{\mathcal{V}}= & \sum_{i=1}^{m} v_{i} \dot{\mathcal{V}}_{i}=\sum_{i=1}^{m} v_{i}\left[\dot{\mathcal{V}}_{h, i}+\sum_{j=1}^{p} w_{i j} \dot{\mathcal{V}}_{v, j}\right] \\
= & \sum_{i=1}^{m} v_{i}[\underbrace{\mu_{i} S_{i}^{*}\left(2-\frac{S_{i}^{*}}{S_{i}}-\frac{S_{i}}{S_{i}^{*}}\right)}_{\mathfrak{A}_{h, i}}+\sum_{j=1}^{p^{\prime}} a_{i, j} \beta_{i, j}^{\diamond} S_{i}^{*} \frac{I_{v, j}^{*}}{N_{i}}\left(3-\frac{S_{i}^{*}}{S_{i}}-\frac{S_{i}}{S_{i}^{*}} \frac{I_{v, j}}{I_{v, j}^{*}} \frac{E_{i}^{*}}{E_{i}}-\frac{I_{i}^{*}}{I_{i}} \frac{E_{i}}{E_{i}^{*}}\right) \\
& +\sum_{j=p^{\prime}+1}^{p} \underbrace{a_{i, j} \beta_{i, j}^{\diamond} S_{i}^{*} \frac{d_{j}}{N_{i}}\left(4-\frac{S_{i}^{*}}{S_{i}}-\frac{S_{i}}{S_{i}^{*}} \frac{E_{i}^{*}}{E_{i}}-\frac{I_{i}^{*}}{I_{i}} \frac{E_{i}}{E_{i}^{*}}-\frac{I_{i}}{I_{i}^{*}}\right)}_{\mathfrak{c}_{i j}}+\sum_{j=1}^{p^{\prime}} a_{i, j} \beta_{i, j}^{\diamond} S_{i}^{*} \frac{I_{v, j}}{N_{i}} \\
& \left.-\sum_{j=1}^{p^{\prime}} a_{i, j} \beta_{i, j}^{\diamond} S_{i}^{*} \frac{I_{v, j}^{*}}{N_{i}}\right) \frac{I_{i}}{I_{i}^{*}}+\sum_{j=1}^{p^{\prime}} \underbrace{\mathfrak{A}_{v, j}}_{w_{i j}\left(\mu_{v, j}+\delta_{v, j}\right) S_{v, j}^{*}\left(2-\frac{S_{v, j}^{*}}{S_{v, j}}-\frac{S_{v, j}}{S_{v, j}^{*}}\right)} \\
& +\sum_{j=1}^{p^{\prime}} w_{i j} \sum_{i=1}^{m^{\prime}} a_{i, j} \beta_{i, j} S_{v, j}^{*} \frac{I_{i}^{*}}{N_{i}}\left(2-\frac{S_{v, j}^{*}}{S_{v, j}}-\frac{I_{v, j}^{*}}{I_{v, j}} \frac{S_{v, j}}{S_{v, j}^{*}} \frac{I_{i}}{I_{i}^{*}}\right) \\
& +\sum_{j=1}^{p^{\prime}} w_{i j} \sum_{i=m^{\prime}+1}^{m} \underbrace{}_{a_{i, j} \beta_{i, j} S_{v, j}^{*} \frac{\tilde{d}_{i}}{N_{i}}\left(3-\frac{S_{v, j}^{*}}{S_{v, j}}-\frac{I_{v, j}^{*}}{I_{v, j}} \frac{S_{v, j}}{S_{v, j}^{*}}-\frac{I_{v, j}}{I_{v, j}^{*}}\right)} \\
& \left.-\sum_{j=1}^{p^{\prime}} w_{i j}\left(\sum_{i=1}^{m^{\prime}} a_{i, j} \beta_{i, j} S_{v, j}^{*} \frac{I_{i}^{*}}{N_{i}}\right) \frac{I_{v, j}}{I_{v, j}^{*}}+\sum_{j=1}^{p^{\prime}} w_{i j} \sum_{i=1}^{m^{\prime}} a_{i, j} \beta_{i, j} S_{v, j}^{*} \frac{I_{i}}{N_{i}}\right]
\end{aligned}
$$


The terms $\mathfrak{A}_{h, i}, \mathfrak{A}_{v, j}, \mathfrak{C}_{i j}$ and $\tilde{\mathfrak{C}}_{i j}$ are negative definite. So, we focus on other terms in (3.11). Moreover, by using the expression of $w_{i j}$ given at the beginning of the proof, the terms in $I_{v, j}$ cancel in (3.11); and thus:

$$
\begin{aligned}
\dot{\mathcal{V}}= & \sum_{i=1}^{m^{\prime}} v_{i}\left[\mathfrak{A}_{h, i}+\sum_{j=p^{\prime}+1}^{p} \mathfrak{C}_{i j}+\sum_{j=1}^{p^{\prime}} \mathfrak{A}_{v, j}+\sum_{j=1}^{p^{\prime}} w_{i j} \sum_{i=m^{\prime}+1}^{m} \tilde{\mathfrak{C}}_{i j}\right. \\
& +\sum_{j=1}^{p^{\prime}} a_{i, j} \beta_{i, j}^{\diamond} S_{i}^{*} \frac{I_{v, j}^{*}}{N_{i}}\left(3-\frac{S_{i}^{*}}{S_{i}}-\frac{S_{i}}{S_{i}^{*}} \frac{I_{v, j}}{I_{v, j}^{*}} \frac{E_{i}^{*}}{E_{i}}-\frac{I_{i}^{*}}{I_{i}} \frac{E_{i}}{E_{i}^{*}}\right)-\left(\sum_{j=1}^{p^{\prime}} a_{i, j} \beta_{i, j}^{\diamond} S_{i}^{*} \frac{I_{v, j}^{*}}{N_{i}}\right) \frac{I_{i}}{I_{i}^{*}} \\
& \left.+\sum_{j=1}^{p^{\prime}} w_{i j} \sum_{i=1}^{m^{\prime}} a_{i, j} \beta_{i, j} S_{v, j}^{*} \frac{I_{i}^{*}}{N_{i}}\left(2-\frac{S_{v, j}^{*}}{S_{v, j}}-\frac{I_{v, j}^{*}}{I_{v, j}} \frac{S_{v, j}}{S_{v, j}^{*}} \frac{I_{i}}{I_{i}^{*}}\right)+\sum_{j=1}^{p^{\prime}} w_{i j} \sum_{i=1}^{m^{\prime}} a_{i, j} \beta_{i, j} S_{v, j}^{*} \frac{I_{i}}{N_{i}}\right]
\end{aligned}
$$

Now, we claim that the linear terms in $I_{i}$ cancel in (3.12). That is:

$$
\sum_{i=1}^{m^{\prime}} v_{i}\left[-\left(\sum_{j=1}^{p^{\prime}} a_{i, j} \beta_{i, j}^{\diamond} S_{i}^{*} \frac{I_{v, j}^{*}}{N_{i}}\right) \frac{I_{i}}{I_{i}^{*}}+\sum_{j=1}^{p^{\prime}} w_{i j} \sum_{i=1}^{m^{\prime}} a_{i, j} \beta_{i, j} S_{v, j}^{*} \frac{I_{i}}{N_{i}}\right]=0 .
$$

or equivalently,

$$
\sum_{i=1}^{m^{\prime}} v_{i}\left[-\left(\sum_{j=1}^{p^{\prime}} a_{i, j} \beta_{i, j}^{\diamond} S_{i}^{*} \frac{I_{v, j}^{*}}{N_{i}}\right) \frac{I_{i}}{I_{i}^{*}}+\sum_{j=1}^{p^{\prime}} w_{i j} \sum_{l=1}^{m^{\prime}} a_{l, j} \beta_{l, j} S_{v, j}^{*} \frac{I_{l}}{N_{l}}\right]=0 .
$$

However, note that:

$$
\begin{aligned}
\sum_{j=1}^{p^{\prime}} w_{i j}\left(\sum_{l=1}^{m^{\prime}} a_{l, j} \beta_{l, j} S_{v, j}^{*} \frac{I_{l}}{N_{l}}\right) & =\sum_{j=1}^{p^{\prime}}\left(\sum_{l=1}^{m^{\prime}} w_{i j} a_{l, j} \beta_{l, j} S_{v, j}^{*} \frac{I_{l}}{N_{l}}\right)=\sum_{l=1}^{m^{\prime}} \underbrace{\left(\sum_{j=1}^{p^{\prime}} w_{i j} a_{l, j} \beta_{l, j} S_{v, j}^{*} \frac{1}{N_{l}}\right)}_{\alpha_{i l}} \\
& =\sum_{l=1}^{m^{\prime}} I_{l} \alpha_{i l} .
\end{aligned}
$$

Hence, by summing over on equation (3.13), we obtain

$$
\begin{aligned}
\sum_{i=1}^{m^{\prime}} v_{i}\left(\sum_{j=1}^{p^{\prime}} w_{i j} \sum_{l=1}^{m^{\prime}} a_{l, j} \beta_{l, j} S_{v, j}^{*} \frac{I_{l}}{N_{l}}\right) & =\sum_{i=1}^{m^{\prime}} v_{i}\left(\sum_{l=1}^{m^{\prime}} I_{l} \alpha_{i l}\right)=\sum_{l=1}^{m^{\prime}} v_{l}\left(\sum_{i=1}^{m^{\prime}} I_{i} \alpha_{l i}\right)=\sum_{i=1}^{m^{\prime}}\left(\sum_{l=1}^{m^{\prime}} v_{l} \alpha_{l i}\right) I_{i} \\
& =\sum_{i=1}^{m^{\prime}}\left(\sum_{l=1}^{m^{\prime}} v_{l} \sum_{j=1}^{p^{\prime}} w_{l j} a_{i, j} \beta_{i, j} S_{v, j}^{*} \frac{I_{i}^{*}}{N_{i}}\right) \frac{I_{i}}{I_{i}^{*}} .
\end{aligned}
$$

Hence, to show $(E)$, we need to show that:

$$
\sum_{i=1}^{m^{\prime}} v_{i}\left(\sum_{j=1}^{p^{\prime}} a_{i, j} \beta_{i, j}^{\diamond} S_{i}^{*} \frac{I_{v, j}^{*}}{N_{i}}\right) \frac{I_{i}}{I_{i}^{*}}=\sum_{i=1}^{m^{\prime}}\left(\sum_{l=1}^{m^{\prime}} v_{l} \sum_{j=1}^{p^{\prime}} w_{l j} a_{i, j} \beta_{i, j} S_{v, j}^{*} \frac{I_{i}^{*}}{N_{i}}\right) \frac{I_{i}}{I_{i}^{*}}
$$


or equivalently,

$$
v_{i} \sum_{j=1}^{p^{\prime}} a_{i, j} \beta_{i, j}^{\diamond} S_{i}^{*} \frac{I_{v, j}^{*}}{N_{i}}=\sum_{l=1}^{m^{\prime}} v_{l} \sum_{j=1}^{p^{\prime}} w_{l j} a_{i, j} \beta_{i, j} S_{v, j}^{*} \frac{I_{i}^{*}}{N_{i}}, \quad \text { for all } \quad i \quad(E)^{\prime} .
$$

The $v_{l}$ are then solutions of $B v=0$ where

$$
B=\left(\begin{array}{ccccc}
\boldsymbol{q}_{11} & \sum_{j=1}^{p^{\prime}} w_{2 j} \frac{a_{1, j} \beta_{1, j} S_{v, j}^{*} I_{1}^{*}}{N_{1}} & \ldots & \sum_{j=1}^{p^{\prime}} w_{m^{\prime} j} \frac{a_{1, j} \beta_{1, j} S_{v, j}^{*} I_{1}^{*}}{N_{1}} \\
\sum_{j=1}^{p^{\prime}} w_{1 j} \frac{a_{2, j} \beta_{2, j} S_{v, j}^{*} I_{2}^{*}}{N_{2}} & \boldsymbol{\beta}_{22} & \ldots & \sum_{j=1}^{p^{\prime}} w_{m^{\prime} j} \frac{a_{2, j} \beta_{2, j} S_{v, j}^{*} I_{2}^{*}}{N_{2}} \\
\vdots & \vdots & \ddots & \vdots \\
\sum_{j=1}^{p^{\prime}} w_{1 j} \frac{a_{m^{\prime}, j} \beta_{m^{\prime}, j} S_{v, j}^{*} I_{m^{\prime}}^{*}}{N_{m^{\prime}}} & \sum_{j=1}^{p^{\prime}} w_{2 j} \frac{a_{m^{\prime}, j} \beta_{m^{\prime}, j} S_{v, j}^{*} I_{m^{\prime}}^{*}}{N_{m^{\prime}}} & \ldots & \boldsymbol{\leftrightarrow}_{m^{\prime} m^{\prime}}
\end{array}\right)
$$

where

$$
\mathbf{q}_{k k}=\sum_{j=1}^{p^{\prime}} w_{k j}\left(-\sum_{i=1, i \neq k}^{m^{\prime}} \frac{a_{i, j} S_{v, j}^{*} \beta_{i, j} I_{i}^{*}}{N_{i}}\right) \text {. }
$$

Hence, Relation $(E)^{\prime}$ is satisfied given that the $v_{l}$ are the components of the solution of $B v=0$. Given the expressions of $w_{i j}$, the entries of $B$, for $i \neq j$, can be written as:

$$
\mathbf{a}_{i j}=\frac{I_{i}^{*}}{N_{i}} \sum_{k=1}^{p^{\prime}} w_{j k} a_{i, k} \beta_{i, k} S_{v, k}^{*}=\frac{I_{i}^{*} S_{j}^{*}}{N_{i} N_{j}} \sum_{k=1}^{p^{\prime}} \frac{a_{j, k} \beta_{j, k}^{\diamond} a_{i, k} \beta_{i, k} I_{v, k}^{*}}{\sum_{l=1}^{m^{\prime}} a_{l, k} \beta_{l, k} \frac{I_{l}^{*}}{N_{l}}},
$$

and $\left(A \circ B^{\diamond} .(A \circ B)^{T}\right)_{i j}=\sum_{k=1}^{p^{\prime}} a_{j, k} \beta_{j, k}^{\diamond} a_{i, k} \beta_{i, k}$, for $i \neq j$.

The component $\mathcal{C}$ is a strongly connected component with $m^{\prime}$ hosts and $p^{\prime}$ vectors. Hence, the matrix $A \circ B^{\diamond}(A \circ B)^{T}$ and $(A \circ B)^{T} A \circ B^{\diamond}$ are irreducible. This implies that, by construction, the matrix $B$ is irreducible (see Relation (3.14)).

The matrix $B$ represents the Laplacian of the network connectivity on $\mathcal{C}$ and thus the positivity of $v$ such that $B v=0$ follows using can be proved using Gershgorin's theorem and Perron-Frobenius Theorem for irreducible Metzler matrices ([32], Chap. 4, Cor. 3.2). Moreover, $v_{i}=C_{i i}$, where $C_{i i}$ are the cofactors of $B$.

Therefore, equation (3.12) reduces to

$$
\begin{aligned}
\dot{\mathcal{V}}= & \sum_{i=1}^{m^{\prime}} v_{i}\left[\mathfrak{A}_{h, i}+\sum_{j=p^{\prime}+1}^{p} \mathfrak{C}_{i j}+\sum_{j=1}^{p^{\prime}} \mathfrak{A}_{v, j}+\sum_{j=1}^{p^{\prime}} w_{i j} \sum_{i=m^{\prime}+1}^{m} \tilde{\mathfrak{C}}_{i j}\right. \\
& +\sum_{j=1}^{p^{\prime}} a_{i, j} \beta_{i, j}^{\diamond} S_{i}^{*} \frac{I_{v, j}^{*}}{N_{i}}\left(3-\frac{S_{i}^{*}}{S_{i}}-\frac{S_{i}}{S_{i}^{*}} \frac{I_{v, j}}{I_{v, j}^{*}} \frac{E_{i}^{*}}{E_{i}}-\frac{I_{i}^{*}}{I_{i}} \frac{E_{i}}{E_{i}^{*}}\right) \\
& \left.+\sum_{j=1}^{p^{\prime}} w_{i j} \sum_{i=1}^{m^{\prime}} a_{i, j} \beta_{i, j} S_{v, j}^{*} \frac{I_{i}^{*}}{N_{i}}\left(2-\frac{S_{v, j}^{*}}{S_{v, j}}-\frac{I_{v, j}^{*}}{I_{v, j}} \frac{S_{v, j}}{S_{v, j}^{*}} \frac{I_{i}}{I_{i}^{*}}\right)\right]
\end{aligned}
$$


To avoid confusion, we switch the index $i$ by $l$ in the third term of the previous equation. Hence,

$$
\begin{aligned}
\dot{\mathcal{V}}= & \sum_{i=1}^{m^{\prime}} v_{i}\left[\mathfrak{A}_{h, i}+\sum_{j=p^{\prime}+1}^{p} \mathfrak{C}_{i j}+\sum_{j=1}^{p^{\prime}} \mathfrak{A}_{v, j}+\sum_{j=1}^{p^{\prime}} w_{i j} \sum_{i=m^{\prime}+1}^{m} \tilde{\mathfrak{C}}_{i j}\right. \\
& +\sum_{j=1}^{p^{\prime}} a_{i, j} \beta_{i, j}^{\diamond} S_{i}^{*} \frac{I_{v, j}^{*}}{N_{i}}\left(3-\frac{S_{i}^{*}}{S_{i}}-\frac{S_{i}}{S_{i}^{*}} \frac{I_{v, j}}{I_{v, j}^{*}} \frac{E_{i}^{*}}{E_{i}}-\frac{I_{i}^{*}}{I_{i}} \frac{E_{i}}{E_{i}^{*}}\right) \\
& \left.+\sum_{j=1}^{p^{\prime}} w_{i j} \sum_{l=1}^{m^{\prime}} a_{l, j} \beta_{l, j} S_{v, j}^{*} \frac{I_{l}^{*}}{N_{l}}\left(2-\frac{S_{v, j}^{*}}{S_{v, j}}-\frac{I_{v, j}^{*}}{I_{v, j}} \frac{S_{v, j}}{S_{v, j}^{*}} \frac{I_{l}}{I_{l}^{*}}\right)\right]
\end{aligned}
$$

By using the expression of $w_{i j},(3.15)$ becomes:

$$
\begin{aligned}
\dot{\mathcal{V}}= & \sum_{i=1}^{m^{\prime}} v_{i}\left[\mathfrak{A}_{h, i}+\sum_{j=p^{\prime}+1}^{p} \mathfrak{C}_{i j}+\sum_{j=1}^{p^{\prime}} \mathfrak{A}_{v, j}+\sum_{j=1}^{p^{\prime}} w_{i j} \sum_{i=m^{\prime}+1}^{m} \tilde{\mathfrak{C}}_{i j}\right] \\
& +\sum_{i=1}^{m^{\prime}} \sum_{j=1}^{p^{\prime}} \sum_{l=1}^{m^{\prime}} v_{i} w_{i j} a_{l, j} \beta_{l, j} S_{v, j}^{*} \frac{I_{l}^{*}}{N_{l}}\left(5-\frac{S_{i}^{*}}{S_{i}}-\frac{S_{i}}{S_{i}^{*}} \frac{I_{v, j}}{I_{v, j}^{*}} \frac{E_{i}^{*}}{E_{i}}-\frac{I_{i}^{*}}{I_{i}} \frac{E_{i}}{E_{i}^{*}}-\frac{S_{v, j}^{*}}{S_{v, j}}-\frac{I_{v, j}^{*}}{I_{v, j}} \frac{S_{v, j}}{S_{v, j}^{*}} \frac{I_{l}}{I_{l}^{*}}\right) \\
= & \sum_{i=1}^{m^{\prime}} v_{i}\left[\mathfrak{A}_{h, i}+\sum_{j=p^{\prime}+1}^{p} \mathfrak{C}_{i j}+\sum_{j=1}^{p^{\prime}} \mathfrak{A}_{v, j}+\sum_{j=1}^{p^{\prime}} w_{i j} \sum_{i=m^{\prime}+1}^{m} \tilde{\mathfrak{C}}_{i j}\right]+H_{m^{\prime}},
\end{aligned}
$$

where

$$
H_{m^{\prime}}:=\sum_{i=1}^{m^{\prime}} \sum_{j=1}^{p^{\prime}} \sum_{l=1}^{m^{\prime}} v_{i} w_{i j} \bar{\beta}_{l, j}\left(5-\frac{S_{i}^{*}}{S_{i}}-\frac{S_{i}}{S_{i}^{*}} \frac{I_{v, j}}{I_{v, j}^{*}} \frac{E_{i}^{*}}{E_{i}}-\frac{I_{i}^{*}}{I_{i}} \frac{E_{i}}{E_{i}^{*}}-\frac{S_{v, j}^{*}}{S_{v, j}}-\frac{I_{v, j}^{*}}{I_{v, j}} \frac{S_{v, j}}{S_{v, j}^{*}} \frac{I_{l}}{I_{l}^{*}}\right),
$$

with $\bar{\beta}_{l, j}=a_{l, j} \beta_{l, j} S_{v, j}^{*} \frac{I_{l}^{*}}{N_{l}}$.

Now, since the matrix $B$ is irreducible, using the arithmetic mean-geometric mean Inequality, we have that $H_{m^{\prime}}$ is definite negative [20]. Hence, it follows that (3.16), as a sum of definite-negative terms, is definite-negative. This ends the proof.

\subsection{Vector-borne models in patchy environments}

Mathematical models that describe the evolution of vector-borne diseases where the environment is structured in patches have been investigated in different settings. However, only recently the global dynamic of these models have been established [11, 24, 29] under some hypotheses. For these models, an irreducibility hypothesis is required to prove the uniqueness and global stability for the interior equilibrium. In [11], numerical simulations have been used to indicate the existence and potential stability of a boundary equilibrium when the mobility matrix is not irreducible. However, a theoretical assessment of the dynamics when the network is reducible has been an open problem. In this subsection, we establish the global asymptotic stability of the equilibria when the network is reducible, using the method described in Section 2. A particular case of this result is that the irreducibility hypothesis is a sufficient but not necessary for the existence of an interior equilibrium. Let us consider a spatially heterogeneous environment with $n$ patches, within which there are human host and mosquito vectors. The host populations travel between patches following a Lagrangian approach [11, 13, 28], for which residence times is used to model human mobility. We consider an SIR-SI epidemiological structure. Particularly, we consider the model: 


$$
\text { for } i=1, \ldots, n,\left\{\begin{array}{l}
\dot{S}_{i}=\Lambda_{h, i}-S_{h, i} \sum_{j=1}^{n} l_{i j} I_{v, j}-\mu_{h, i} S_{h, i} \\
\dot{I}_{h, i}=S_{h, i} \sum_{j=1}^{n} l_{i j} I_{v, j}-\left(\mu_{h, i}+\gamma_{h, i}\right) I_{h, i} \\
\dot{S}_{v, i}=\Lambda_{v, i}-S_{v, i} \sum_{j=1}^{n} m_{i j} I_{h, j}-\left(\mu_{v, i}+\delta_{v, i}\right) S_{v, i} \\
\dot{I}_{v, i}=S_{v, i} \sum_{j=1}^{n} m_{i j} I_{h, j}-\left(\mu_{v, i}+\delta_{v, i}\right) I_{v, i},
\end{array}\right.
$$

This model is equivalent to the models considered in $[11,24]$. In this case, the matrices $M=\left(m_{i j}\right)$ and $L=\left(l_{i j}\right)$ are compound and the values of their entries represent a complex biological cascade of events leading to the infection, including the biting rate per patches, probability of infectiousness, infection force and the mobility patterns of the hosts. For convenience, we recall the results when the connectivity matrix is irreducible. In this case, the model has a sharp threshold property whether the disease either dies out from all patches or persists in all of them depending on whether the threshold known as the basic reproduction number is below or above unity. The basic reproduction number of System (3.17) is:

$$
\mathcal{R}_{0}^{2}=\rho\left(\operatorname{diag}\left(\mathbf{S}_{v}^{0}\right) M \operatorname{diag}^{-1}\left(\mu_{h}+\gamma_{h}\right) \operatorname{diag}\left(\mathbf{S}_{h}^{0}\right) L \operatorname{diag}^{-1}\left(\mu_{v}+\delta\right)\right),
$$

where

$$
\mathbf{S}_{h}^{0}=\operatorname{diag}^{-1}\left(\mu_{h}\right) \boldsymbol{\Lambda}_{h}, \mathbf{S}_{v}^{0}=\operatorname{diag}^{-1}\left(\mu_{v}+\delta\right) \boldsymbol{\Lambda}_{v}, M=\left(m_{i j}\right), L=\left(l_{i j}\right),
$$

and $\rho($.$) the spectral radius operator.$

Theorem 3.6. [11, 24] Under the assumption that the network is irreducible, we have that

1. If $\mathcal{R}_{0} \leq 1$, the DFE is globally asymptotically stable.

2. If $\mathcal{R}_{0}>1$, the DFE is unstable and it exists a unique interior equilibrium that is GAS.

Now, our goal is to investigate the dynamics when the network is reducible. In this case, we can re-structure the network into smaller irreducible components. The components could be either maximal (i.e., sink) or minimal (i.e., source). If the component is minimal, then its dynamics is not influenced by the rest of the network and its dynamics is equivalent to that of Theorem 3.6. Let $\mathcal{C}$, an irreducible component that has $n^{\prime}$ patches (with $\left.n^{\prime} \leq n\right)$ and that is maximal. Let us consider the dynamics of the auxiliary system on that component:

$$
\text { for } i=1 \ldots n^{\prime},\left\{\begin{array}{l}
\dot{S}_{i}=\Lambda_{h, i}-S_{h, i} \sum_{j=1}^{n^{\prime}} l_{i j} I_{v, j}-S_{h, i} \sum_{j=n^{\prime}+1}^{n} l_{i j} d_{j}-\mu_{h, i} S_{h, i} \\
\dot{I}_{h, i}=S_{h, i} \sum_{j=1}^{n^{\prime}} l_{i j} I_{v, j}+S_{h, i} \sum_{j=n^{\prime}+1}^{n} l_{i j} d_{j}-\left(\mu_{h, i}+\gamma_{h, i}\right) I_{h, i} \\
\dot{S}_{v, i}=\Lambda_{v, i}-S_{v, i} \sum_{j=1}^{n^{\prime}} m_{i j} I_{h, j}-S_{v, i} \sum_{j=n^{\prime}+1}^{n} m_{i j} \tilde{d}_{j}-\left(\mu_{v, i}+\delta_{v, i}\right) S_{v, i} \\
\dot{I}_{v, i}=S_{v, i} \sum_{j=1}^{n^{\prime}} m_{i j} I_{h, j}+S_{v, i} \sum_{j=n^{\prime}+1}^{n} m_{i j} \tilde{d}_{j}-\left(\mu_{v, i}+\delta_{v, i}\right) I_{v, i}
\end{array}\right.
$$


Theorem 3.7. Let $\boldsymbol{x}^{*}$ an equilibrium for Model (3.18). Then, $\boldsymbol{x}^{*}$ is GAS for all $(\tilde{d}, d) \geq 0$. Particularly, $\boldsymbol{x}^{*}$ is an interior equilibrium if $\mathcal{R}_{0, \mathcal{C}}>1$ or if $(\tilde{d}, d) \gg 0$.

The equilibrium $\boldsymbol{x}^{*}$ can be a boundary or an interior equilibrium depending on its $\mathcal{R}_{0, \mathcal{C}}$ and the equilibrium of states of the other irreducible components affecting the dynamics on $\mathcal{C}$. That is, on the value of the vector $(\tilde{d}, d)$. If $(\tilde{d}, d):=0$, System (3.18) has the same dynamics as System (3.17), which are provided by Theorem 3.6. If $(\tilde{d}, d) \gg 0$, the trajectories of System (3.18) converge toward an interior equilibrium, regardless of the value of the basic reproduction number of System (3.18).

Proof of Theorem 3.7. Let us consider the following Lyapunov candidate:

$$
V=\sum_{i=1}^{n^{\prime}} g_{h, i}\left(S_{h, i}-S_{h, i}^{*} \ln S_{h, i}+I_{h, i}-I_{h, i}^{*} \ln I_{h, i}\right)+\sum_{i=1}^{n^{\prime}} g_{v, i}\left(S_{v, i}-S_{v, i}^{*} \ln S_{v, i}+I_{v, i}-I_{v, i}^{*} \ln I_{v, i}\right)
$$

where $g_{v, k}$, for $k=1, \ldots, n^{\prime}$ are given by

$$
g_{v, k}=\frac{1}{S_{v, k}^{*} \sum_{j=1}^{n^{\prime}} m_{k j} I_{h, j}^{*}} \sum_{i=1}^{n^{\prime}} g_{h, i} S_{h, i}^{*} l_{i k} I_{v, k}^{*}
$$

Since $\mathcal{C}$ is an irreducible component with $n^{\prime}$ patches, the coefficients $g_{v, k}$ are well-defined. The coefficients $g_{h, i}$, for $i=1,2, \ldots, n^{\prime}$ will be defined later. The derivative of $V$ along the trajectories of System (3.18) is given by:

$$
\begin{aligned}
\dot{V}= & A_{h}+A_{v}+\sum_{i=1}^{n^{\prime}} g_{h, i}\left(S_{h, i}^{*} \sum_{j=n^{\prime}+1}^{n} l_{i j} d_{j}\right)\left(3-\frac{S_{h, i}}{S_{h, i}^{*}}-\frac{S_{h, i}}{S_{h, i}^{*}} \frac{I_{h, i}^{*}}{I_{h, i}}-\frac{I_{h, i}}{I_{h, i}^{*}}\right) \\
& +\sum_{i=1}^{n^{\prime}} g_{h, i} S_{h, i}^{*} \sum_{j=1}^{n^{\prime}} l_{i j} I_{v, j}^{*}\left(1-\frac{S_{h, i}^{*}}{S_{h, i}}\right)+\sum_{i=1}^{n^{\prime}} g_{h, i} S_{h, i}^{*} \sum_{j=1}^{n^{\prime}} l_{i j} I_{v, j} \\
& -\sum_{i=1}^{n^{\prime}} g_{h, i} \frac{I_{h, i}}{I_{h, i}^{*}}\left(S_{h, i}^{*} \sum_{j=1}^{n^{\prime}} l_{i j} I_{v, j}^{*}\right)-\sum_{i=1}^{n^{\prime}} g_{h, i} \frac{I_{h, i}^{*}}{I_{h, i}}\left(S_{h, i} \sum_{j=1}^{n^{\prime}} l_{i j} I_{v, j}\right)+\sum_{i=1}^{n^{\prime}} g_{h, i}\left(S_{h, i}^{*} \sum_{j=1}^{n^{\prime}} l_{i j} I_{v, j}^{*}\right) . \\
& +\sum_{i=1}^{n^{\prime}} g_{v, i} S_{v, i}^{*} \sum_{j=n^{\prime}+1}^{n} m_{i j} \tilde{d}_{j}\left(3-\frac{S_{v, i}^{*}}{S_{v, i}}-\frac{S_{v, i}}{S_{v, i}^{*}} \frac{I_{v, i}^{*}}{I_{v, i}}-\frac{I_{v, i}}{I_{v, i}^{*}}\right)+\sum_{i=1}^{n^{\prime}} g_{v, i} S_{v, i}^{*} \sum_{j=1}^{n^{\prime}} m_{i j} I_{h, j} \\
& -\sum_{i=1}^{n^{\prime}} g_{v, i} \frac{I_{v, i}}{I_{v, i}^{*}}\left(S_{v, i}^{*} \sum_{j=1}^{n^{\prime}} m_{i j} I_{h, j}^{*}\right)+\sum_{i=1}^{n^{\prime}} g_{v, i} S_{v, i}^{*} \sum_{j=1}^{n^{\prime}} m_{i j} I_{h, j}^{*}\left(2-\frac{S_{v, i}^{*}}{S_{v, i}}-\frac{S_{v, i}}{S_{v, i}^{*}} \frac{I_{v, i}^{*}}{I_{v, i}} \frac{I_{h, j}}{I_{h, j}^{*}}\right)
\end{aligned}
$$

where $A_{v}=\sum_{i=1}^{n^{\prime}} g_{v, i}\left(\mu_{v, i}+\delta_{v, i}\right) S_{v, i}^{*}\left(2-\frac{S_{v, i}^{*}}{S_{v, i}}-\frac{S_{v, i}}{S_{v, i}^{*}}\right)$ and $A_{h}=\sum_{i=1}^{n^{\prime}} g_{h, i} \mu_{h, i} S_{h, i}^{*}\left(2-\frac{S_{h, i}^{*}}{S_{h, i}}-\frac{S_{h, i}}{S_{h, i}^{*}}\right)$.

Now, given the expression of $g_{v, i}$, equation (3.19) can be written as:

$$
\dot{V}=A_{h}+A_{v}+\sum_{i=1}^{n^{\prime}} g_{h, i}\left(S_{h, i}^{*} \sum_{j=n^{\prime}+1}^{n} l_{i j} d_{j}\right)\left(3-\frac{S_{h, i}}{S_{h, i}^{*}}-\frac{S_{h, i}}{S_{h, i}^{*}} \frac{I_{h, i}^{*}}{I_{h, i}}-\frac{I_{h, i}}{I_{h, i}^{*}}\right)
$$




$$
\begin{aligned}
& +\sum_{i=1}^{n^{\prime}} g_{h, i} S_{h, i}^{*} \sum_{j=1}^{n^{\prime}} l_{i j} I_{v, j}^{*}\left(2-\frac{S_{h, i}^{*}}{S_{h, i}}-\frac{S_{h, i}}{S_{h, i}^{*}} \frac{I_{h, i}^{*}}{I_{h, i}} \frac{I_{v, j}}{I_{v, j}^{*}}\right)-\sum_{i=1}^{n^{\prime}} g_{h, i} \frac{I_{h, i}}{I_{h, i}^{*}}\left(S_{h, i}^{*} \sum_{j=1}^{n^{\prime}} l_{i j} I_{v, j}^{*}\right) \\
& +\sum_{i=1}^{n^{\prime}} g_{v, i} S_{v, i}^{*} \sum_{j=n^{\prime}+1}^{n} m_{i j} \tilde{d}_{j}\left(3-\frac{S_{v, i}^{*}}{S_{v, i}}-\frac{S_{v, i}}{S_{v, i}^{*}} \frac{I_{v, i}^{*}}{I_{v, i}}-\frac{I_{v, i}}{I_{v, i}^{*}}\right)+\sum_{i=1}^{n^{\prime}} g_{v, i} S_{v, i}^{*} \sum_{j=1}^{n^{\prime}} m_{i j} I_{h, j} \\
& +\sum_{i=1}^{n^{\prime}} g_{v, i} S_{v, i}^{*} \sum_{j=1}^{n^{\prime}} m_{i j} I_{h, j}^{*}\left(2-\frac{S_{v, i}^{*}}{S_{v, i}}-\frac{S_{v, i}}{S_{v, i}^{*}} \frac{I_{v, i}^{*}}{I_{v, i}} \frac{I_{h, j}}{I_{h, j}^{*}}\right) .
\end{aligned}
$$

We choose $g_{h}=\left(g_{h, 1}, g_{h, 2}, \ldots, g_{h, n^{\prime}}\right)^{T}$ to be solution of the system $B g_{h}=0$, where:

$$
B=\left(\begin{array}{cccc}
-\sum_{j=2}^{n^{\prime}} b_{1 j} & b_{21} & \ldots & b_{n^{\prime} 1} \\
b_{12} & -\sum_{j=1, j \neq 2}^{n^{\prime}} b_{2 j} & \ldots & b_{n^{\prime} 2} \\
\vdots & \vdots & \ddots & \vdots \\
b_{1 n^{\prime}} & b_{2 n^{\prime}} & \ldots & -\sum_{j=1}^{n^{\prime}-1} b_{n^{\prime} j}
\end{array}\right)
$$

where, for $1 \leq r, k \leq n^{\prime}$ and $r \neq k$, we have:

$$
b_{r k}=S_{h, r}^{*} \sum_{i=1}^{n^{\prime}} l_{r i} I_{v, i}^{*} \frac{m_{i k} I_{h, k}^{*}}{\sum_{j=1}^{n^{\prime}} m_{i j} I_{h, j}^{*}} .
$$

Since $\mathcal{C}$ is irreducible, then the matrix $B$ is also irreducible, and therefore the system $B g_{h}=0$ has a positive solution $g_{h} \gg 0$. Particularly, for any $k \in\left\{1,2, \ldots, n^{\prime}\right\}, g_{h, k}$ satisfies the relationship

$$
-g_{h, k} S_{h, k}^{*} \sum_{i=1}^{n^{\prime}} l_{k i} I_{v, i}^{*}\left(\frac{\sum_{j=1, j \neq k}^{n^{\prime}} m_{i j} I_{h, j}^{*}}{\sum_{j=1}^{n^{\prime}} m_{i j} I_{h, j}^{*}}\right)+\sum_{r=1, r \neq k}^{n^{\prime}} g_{h, r} S_{h, r}^{*} \sum_{i=1}^{n^{\prime}} l_{r i} I_{v, i}^{*} \frac{m_{i k} I_{h, k}^{*}}{\sum_{j=1}^{n^{\prime}} m_{i j} I_{h, j}^{*}}=0 .
$$

By using the expression $g_{v, i}$, equation (3.21) can be written as:

$$
-g_{h, j} S_{h, j}^{*} \sum_{l=1}^{n^{\prime}} l_{j l} I_{v, l}^{*}+\sum_{i=1}^{n^{\prime}} g_{v, i} S_{v, i}^{*} m_{i j} I_{h, j}^{*}=0 .
$$

Therefore, equation (3.20) leads to:

$$
\begin{aligned}
\dot{V}= & A_{h}+A_{v}+\sum_{i=1}^{n^{\prime}} g_{h, i}\left(S_{h, i}^{*} \sum_{j=n^{\prime}+1}^{n} l_{i j} d_{j}\right)\left(3-\frac{S_{h, i}}{S_{h, i}^{*}}-\frac{S_{h, i}}{S_{h, i}^{*}} \frac{I_{h, i}^{*}}{I_{h, i}}-\frac{I_{h, i}}{I_{h, i}^{*}}\right) \\
& +\sum_{i=1}^{n^{\prime}} g_{v, i} S_{v, i}^{*} \sum_{j=n^{\prime}+1}^{n} m_{i j} \tilde{d}_{j}\left(3-\frac{S_{v, i}^{*}}{S_{v, i}}-\frac{S_{v, i}}{S_{v, i}^{*}} \frac{I_{v, i}^{*}}{I_{v, i}}-\frac{I_{v, i}}{I_{v, i}^{*}}\right)
\end{aligned}
$$




$$
\begin{aligned}
& +\sum_{i=1}^{n^{\prime}} g_{h, i} S_{h, i}^{*} \sum_{j=1}^{n^{\prime}} l_{i j} I_{v, j}^{*}\left(2-\frac{S_{h, i}^{*}}{S_{h, i}}-\frac{S_{h, i}}{S_{h, i}^{*}} \frac{I_{h, i}^{*}}{I_{h, i}} \frac{I_{v, j}}{I_{v, j}^{*}}\right) \\
& +\sum_{i=1}^{n^{\prime}} g_{v, i} S_{v, i}^{*} \sum_{j=1}^{n^{\prime}} m_{i j} I_{h, j}^{*}\left(2-\frac{S_{v, i}^{*}}{S_{v, i}}-\frac{S_{v, i}}{S_{v, i}^{*}} \frac{I_{v, i}^{*}}{I_{v, i}} \frac{I_{h, j}}{I_{h, j}^{*}}\right)
\end{aligned}
$$

The first four terms of (3.22) are definite-negative. Let us denote by $N$ the last two sums in (3.22). By replacing $g_{v, i}$ by its expression and switching the indices $l$ and $i$, we obtain:

$$
\begin{aligned}
N= & \sum_{i=1}^{n^{\prime}} g_{h, i} S_{h, i}^{*} \sum_{j=1}^{n^{\prime}} l_{i j} I_{v, j}^{*}\left(2-\frac{S_{h, i}^{*}}{S_{h, i}}-\frac{S_{h, i}}{S_{h, i}^{*}} \frac{I_{h, i}^{*}}{I_{h, i}} \frac{I_{v, j}}{I_{v, j}^{*}}\right) \\
& +\sum_{l=1}^{n^{\prime}} \frac{1}{\sum_{j=1}^{n^{\prime}} m_{l j} I_{h, j}^{*}} \sum_{i=1}^{n^{\prime}} g_{h, i} S_{h, i}^{*} l_{i l} I_{v, l}^{*} \sum_{j=1}^{n^{\prime}} m_{l j} I_{h, j}^{*}\left(2-\frac{S_{v, l}^{*}}{S_{v, l}}-\frac{S_{v, l}}{S_{v, l}^{*}} \frac{I_{v, l}^{*}}{I_{v, l}} \frac{I_{h, j}}{I_{h, j}^{*}}\right) \\
= & \sum_{i=1}^{n^{\prime}} g_{h, i} S_{h, i}^{*} \sum_{j=1}^{n^{\prime}} l_{i j} I_{v, j}^{*}\left[\left(2-\frac{S_{h, i}^{*}}{S_{h, i}}-\frac{S_{h, i}}{S_{h, i}^{*}} \frac{I_{h, i}^{*}}{I_{h, i}} \frac{I_{v, j}}{I_{v, j}^{*}}\right)\right. \\
& \left.+\frac{1}{\sum_{r=1}^{n^{\prime}} m_{j r} I_{h, r}^{*}} \sum_{k=1}^{n^{\prime}} m_{j k} I_{h, k}^{*}\left(2-\frac{S_{v, j}^{*}}{S_{v, j}}-\frac{S_{v, j}}{S_{v, j}^{*}} \frac{I_{v, j}^{*}}{I_{v, j}} \frac{I_{h, k}}{I_{h, k}^{*}}\right)\right] \\
= & \sum_{i=1}^{n^{\prime}} g_{h, i} S_{h, i}^{*} \sum_{j=1}^{n^{\prime}} l_{i j} I_{v, j}^{*} \frac{\sum_{k=1}^{n^{\prime}} m_{j k} I_{h, k}^{*}}{\sum_{r=1}^{n^{\prime}} m_{j r} I_{h, r}^{*}}\left(4-\frac{S_{h, i}^{*}}{S_{h, i}}-\frac{S_{h, i}}{S_{h, i}^{*}} \frac{I_{h, i}^{*}}{I_{h, i}} \frac{I_{v, j}}{I_{v, j}^{*}}-\frac{S_{v, j}^{*}}{S_{v, j}}-\frac{S_{v, j}}{S_{v, j}^{*}} \frac{I_{v, j}^{*}}{I_{v, j}} \frac{I_{h, k}}{I_{h, k}^{*}}\right),
\end{aligned}
$$

which is definite-positive since the network with $n^{\prime}$ patches is irreducible. This implies $\dot{V}$ is definite-negative and therefore the equilibrium $\boldsymbol{x}^{*}$ is GAS that ends the proof.

Theorem 3.7 provides the global dynamics of the auxiliary system (3.18) and therefore by using Theorem 2.1, a complete global characterization of equilibrium of System (3.17) is obtained, regardless of the topology of the connectivity matrix.

\section{Illustrations}

In this section, we illustrate the method provided in Section 2 and Section 3.1 with a particular case and provide some numerical simulations to highlight the previous results.

\subsection{Case study}

We consider a model with 7 hosts and 7 vectors where the host-vector network configuration is captured by Figure 2. For this case, Model (3.1), disregarding the equations of $\boldsymbol{R}$ that do not impact the remaining 
equations, can be written as:

$$
\left\{\begin{array}{l}
\dot{\boldsymbol{S}}=\boldsymbol{\Lambda}_{h}-\operatorname{diag}^{-1}\left(\overline{\boldsymbol{N}}_{h}\right) \operatorname{diag}(\boldsymbol{S}) A \circ B^{\diamond} \boldsymbol{I}_{v}-\operatorname{diag}(\mu) \boldsymbol{S} \\
\dot{\boldsymbol{E}}=\operatorname{diag}^{-1}\left(\overline{\boldsymbol{N}}_{h}\right) \operatorname{diag}(\boldsymbol{S}) A \circ B^{\diamond} \boldsymbol{I}_{v}-\operatorname{diag}(\mu+\nu) \boldsymbol{E} \\
\dot{\boldsymbol{I}}=\operatorname{diag}(\nu) \boldsymbol{E}-\operatorname{diag}(\alpha) \boldsymbol{I}_{h} \\
\dot{\boldsymbol{S}}_{v}=\boldsymbol{\Lambda}_{v}-\operatorname{diag}\left(\boldsymbol{S}_{v}\right)(A \circ B)^{T} \operatorname{diag}^{-1}\left(\overline{\boldsymbol{N}}_{h}\right) \boldsymbol{I}_{h}-\operatorname{diag}\left(\mu_{v}+\delta_{v}\right) \boldsymbol{S}_{v} \\
\dot{\boldsymbol{I}}_{v}=\operatorname{diag}\left(\boldsymbol{S}_{v}\right)(A \circ B)^{T} \operatorname{diag}^{-1}\left(\overline{\boldsymbol{N}}_{h}\right) \boldsymbol{I}_{h}-\operatorname{diag}\left(\mu_{v}+\delta_{v}\right) \boldsymbol{I}_{v}
\end{array}\right.
$$

where

$$
B^{\diamond}=\left(\begin{array}{ccccccc}
\beta_{1,1}^{\diamond} & 0 & 0 & 0 & 0 & 0 & 0 \\
\beta_{2,1}^{\diamond} & \beta_{2,2}^{\diamond} & 0 & 0 & 0 & 0 & 0 \\
\beta_{3,1}^{\diamond} & 0 & \beta_{3,3}^{\diamond} & \beta_{3,4}^{\diamond} & 0 & 0 & 0 \\
0 & 0 & 0 & 0 & \beta_{4,5}^{\diamond} & 0 & 0 \\
0 & 0 & 0 & 0 & \beta_{5,5}^{\diamond} & 0 & 0 \\
0 & 0 & 0 & 0 & 0 & \beta_{6,6}^{\diamond} & 0 \\
0 & 0 & 0 & 0 & 0 & 0 & \beta_{7,7}^{\diamond}
\end{array}\right), B=\left(\begin{array}{ccccccc}
\beta_{1,1} & 0 & 0 & 0 & 0 & \beta_{1,6} & 0 \\
\beta_{2,1} & \beta_{2,2} & 0 & 0 & 0 & 0 & 0 \\
0 & 0 & \beta_{3,3} & \beta_{3,4} & 0 & 0 & 0 \\
0 & 0 & 0 & 0 & \beta_{4,5} & 0 & 0 \\
0 & 0 & 0 & 0 & \beta_{5,5} & 0 & 0 \\
0 & 0 & 0 & 0 & 0 & \beta_{6,6} & 0 \\
0 & 0 & 0 & 0 & 0 & \beta_{7,6} & \beta_{7,7}
\end{array}\right) .
$$

Assuming the elements of the biting matrix $A$ are positive, it could be shown that the matrices $(A \circ B)^{T} A \circ B^{\diamond}$ and $A \circ B^{\diamond}(A \circ B)^{T}$ are not irreducible. However, as stated earlier, we can structure the network into irreducible components as in Figure 2. Moreover, we can notice from Figure 2 (or the condensed digraph Fig. 3), that $\mathcal{C}_{1}$ and $\mathcal{C}_{5}$ are sources of the condensed graph. That is they are nodes without incoming edges. Thus, the dynamics in these components are independent of variables other than their own. We perform the study on these two sources $\mathcal{C}_{1}$ and $\mathcal{C}_{5}$ :

- On $\mathcal{C}_{1}$, the dynamics is given by, for $i=1,2$ and $j=1,2$,

$$
\left\{\begin{array}{l}
\dot{S}_{i}=\Lambda_{i}-\sum_{j=1}^{2} a_{i, j} \beta_{i, j}^{\diamond} S_{i} \frac{I_{v, j}}{N_{i}}-\mu_{i} S_{i} \\
\dot{E}_{i}=\sum_{j=1}^{2} a_{i, j} \beta_{i, j}^{\diamond} S_{i} \frac{I_{v, j}}{N_{i}}-\left(\mu_{i}+\nu_{i}\right) E_{i} \\
\dot{I}_{i}=\nu_{i} E_{i}-\alpha_{i} I_{i} \\
\dot{S}_{v, j}=\Lambda_{v, j}-\sum_{i=1}^{2} a_{i, j} \beta_{i, j} S_{v, j} \frac{I_{i}}{N_{i}}-\left(\mu_{v, j}+\delta_{v, j}\right) S_{v, j} \\
\dot{I}_{v, j}=\sum_{i=1}^{2} a_{i, j} \beta_{i, j} S_{v, j} \frac{I_{i}}{N_{i}}-\left(\mu_{v, j}+\delta_{v, j}\right) I_{v, j}
\end{array}\right.
$$

The basic reproduction number of Model $C_{1}$ is $\mathcal{R}_{0, \mathcal{C}_{1}}^{2}=\rho\left(\mathcal{N}_{\mathcal{C}_{1}}\right)$ where

$$
\mathcal{N}_{\mathcal{C}_{1}}:=\left(\begin{array}{cc}
\frac{N_{v, 1}}{\mu_{v, 1}+\delta_{v, 1}}\left(\frac{a_{11}^{2} \beta_{1,1} \beta_{1,1}^{\diamond} \nu_{1}}{\left(\nu_{1}+\mu_{1}\right) \alpha_{1} N_{1}}+\frac{a_{21}^{2} \beta_{2,1} \beta_{2,1}^{\diamond} \nu_{2}}{\left(\nu_{2}+\mu_{2}\right) \alpha_{2} N_{2}}\right) & \frac{N_{v, 1}}{\mu_{v, 2}+\delta_{v, 2}}\left(\frac{a_{11} a_{1,2} \beta_{1,1} \beta_{1,2} \nu_{1}}{\left(\nu_{1}+\mu_{1}\right) \alpha_{1} N_{1}}+\frac{a_{21} a_{2,2} \beta_{2,1} \beta_{2,2} \nu_{2}}{\left(\nu_{2}+\mu_{2}\right) \alpha_{2} N_{2}}\right) \\
\frac{N_{v, 2}}{\mu_{v, 1}+\delta_{v, 1}}\left(\frac{a_{1,1} a_{1,2} \beta_{1,2} \beta_{1,1}^{\diamond} \nu_{1}}{\left(\nu_{1}+\mu_{1}\right) \alpha_{1} N_{1}}+\frac{a_{21} a_{2,2} \beta_{2,2} \beta_{2,1}^{\diamond} \nu_{2}}{\left(\nu_{2}+\mu_{2}\right) \alpha_{2} N_{2}}\right) & \frac{N_{v, 2}}{\mu_{v, 2}+\delta_{v, 2}}\left(\frac{a_{12}^{2} \beta_{1,2} \beta_{1,2}^{\circ} \nu_{1}}{\left(\nu_{1}+\mu_{1}\right) \alpha_{1} N_{1}}+\frac{a_{22}^{2} \beta_{2,2} \beta_{2,2}^{\circ} \nu_{2}}{\left(\nu_{2}+\mu_{2}\right) \alpha_{2} N_{2}}\right)
\end{array}\right)
$$


- On $\mathcal{C}_{5}$, the dynamics is given by:

$$
\left\{\begin{array}{l}
\dot{S}_{7}=\Lambda_{7}-a_{1,7} \beta_{1,7}^{\diamond} S_{7} \frac{I_{v, 7}}{N_{7}}-\mu_{7} S_{7} \\
\dot{E}_{7}=a_{7,7} \beta_{7,7}^{\diamond} S_{7} \frac{I_{v, 7}}{N_{7}}-\left(\mu_{7}+\nu_{7}\right) E_{h} \\
\dot{I}_{7}=\nu_{7} E_{7}-\alpha_{7} I_{7} \\
\dot{S}_{v, 7}=\Lambda_{v, 7}-a_{7,7} \beta_{7,7} S_{v, 7} \frac{I_{7}}{N_{7}}-\left(\mu_{v, 7}+\delta_{v, 7}\right) S_{v, 7} \\
\dot{I}_{v, 7}=a_{7,7} \beta_{7,7} S_{v, 7} \frac{I_{7}}{N_{7}}-\left(\mu_{v, 7}+\delta_{v, 7}\right) I_{v, 7}
\end{array}\right.
$$

The basic reproduction number for the irreducible component $\mathcal{C}_{5}$ is:

$$
\mathcal{R}_{0, \mathcal{C}_{5}}^{2}=\frac{a_{7,7}^{2} \beta_{7,7} \beta_{7,7}^{\diamond} \nu_{7} N_{v, 7}}{\left(\delta_{v, 7}+\mu_{v, 7}\right)\left(\mu_{7}+\nu_{7}\right) \alpha_{7} N_{7}} .
$$

Remark 4.1. If $\mathcal{R}_{0, \mathcal{C}_{1}}^{2}>1$ and $\mathcal{R}_{0, \mathcal{C}_{5}}^{2}>1$, then we could anticipate that the endemic equilibrium in the whole network is strongly endemic.

Suppose that $\mathcal{R}_{0, \mathcal{C}_{1}}^{2}<1$ and $\mathcal{R}_{0, \mathcal{C}_{5}}^{2}>1$. Hence, the trajectories of Model $\left(C_{1}\right)$ converges to the $D F E_{\mathcal{C}_{1}}$, which we denote by $\boldsymbol{x}_{1}^{0}=\left(S_{1}^{0}, S_{2}^{0}, 0,0,0,0, S_{v, 1}, S_{v, 2}, 0,0\right)$. Similarly, a unique GAS endemic equilibrium $\overline{\boldsymbol{x}}_{5}=$ $\left(\bar{S}_{7}, \bar{E}_{7}, \bar{I}_{7}, \bar{S}_{v, 7}, \bar{S}_{v, 7}\right)$ for Model $\left(C_{5}\right)$.

Thus, applying the reduction method of Section 2, the dynamics of System 4.1 is equivalent to the model represented by the network illustrated in Figure 4 . Given the dynamics on $\mathcal{C}_{1}$ and $\mathcal{C}_{5}$, the dynamics on the the remaining irreducible component is as follows:

- On $\mathcal{C}_{4}$, the dynamics is given by:

$$
\left\{\begin{array}{l}
\dot{S}_{6}=\Lambda_{6}-a_{6,6} \beta_{6,6}^{\diamond} S_{6} \frac{I_{v, 6}}{N_{6}}-\mu_{6} S_{6} \\
\dot{E}_{6}=a_{6,6} \beta_{6,6}^{\diamond} S_{6} \frac{I_{v, 6}}{N_{6}}-\left(\mu_{6}+\nu_{6}\right) E_{6} \\
\dot{I}_{6}=\nu_{6} E_{6}-\alpha_{6} I_{6} \\
\dot{S}_{v, 6}=\Lambda_{v, 6}-a_{6,6} \beta_{6,6} S_{v, 6} \frac{I_{6}}{N_{6}}-a_{7,6} \beta_{7,6} S_{v, 6} \frac{\bar{I}_{7}}{N_{6}}-\left(\mu_{v, 6}+\delta_{v, 6}\right) S_{v, 6} \\
\dot{I}_{v, 6}=a_{6,6} \beta_{6,6} S_{v, 6} \frac{I_{6}}{N_{6}}+a_{7,6} \beta_{7,6} S_{v, 6} \frac{\bar{I}_{7}}{N_{6}}-\left(\mu_{v, 6}+\delta_{v, 6}\right) I_{v, 6}
\end{array}\right.
$$

since $I_{v, 1}^{0}=0$ (the infected vector at equilibrium from $\mathcal{C}_{1}$ ). Using Remark 3.4, the basic reproduction number on $C_{4}$ is:

$$
\mathcal{R}_{0, \mathcal{C}_{4}}^{2}=\frac{a_{6,6}^{2} \beta_{6,6} \beta_{6,6}^{\diamond} \nu_{6} N_{v, 6}}{\alpha_{6}\left(\mu_{6}+\nu_{6}\right)\left(\delta_{v, 6}+\mu_{v, 6}\right) N_{6}} .
$$

However, it is worthwhile noticing that, regardless of the values of $\mathcal{R}_{0, \mathcal{C}_{4}}^{2}$, the disease will be endemic in this component. That is because the system behaves as if there is a continuous influx of infected $\left(\bar{I}_{7} \gg 0\right)$ into the system (see [7]). Moreover, System $\left(C_{4}\right)$ has the same form as System (3.3) given in Theorem 3.3, where $d_{7}=0$ and $\tilde{d}_{7}=\bar{I}_{7}$. Thus, according to Theorem 3.3, the unique endemic equilibrium of Model $\left(C_{4}\right), \overline{\boldsymbol{x}}_{4}=$ $\left(\bar{S}_{6}, \bar{E}_{6}, \bar{I}_{6}, \bar{S}_{v, 6}, \bar{S}_{v, 6}\right)$, is GAS. Moreover, the component $\mathcal{C}_{4}$ is decoupled from the rest of the system (see Fig. 4 ). Thus, it remains to study the system given by the following network (Fig. 5): 


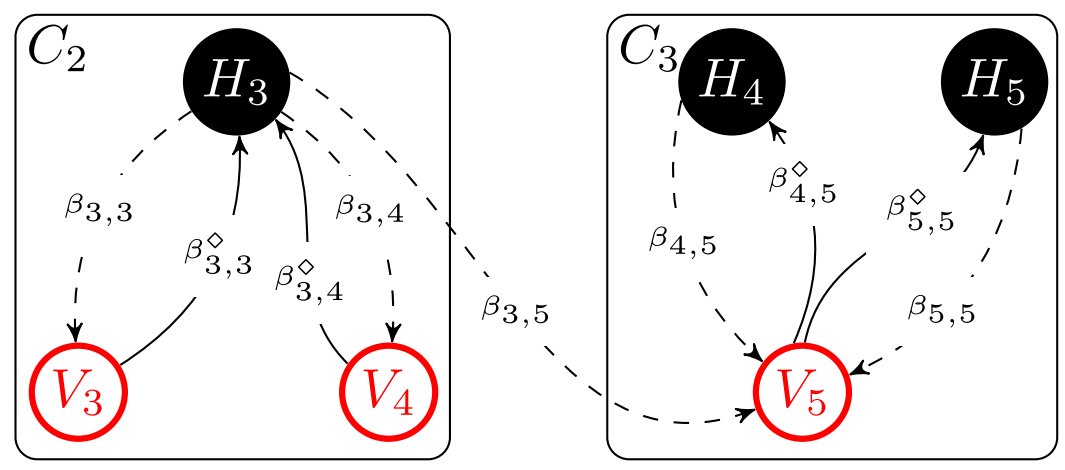

FiguRE 5. Connected part of Figure 4.

Now, we apply the reduction method on the network configuration of Figure 5. Indeed, in Figure $5, \mathcal{C}_{2}$ is the minimal element and the dynamics on it is given by:

$$
\left\{\begin{array}{l}
\dot{S}_{3}=\Lambda_{3}-\sum_{j=3}^{4} a_{3, j} \beta_{3, j}^{\diamond} S_{3} \frac{I_{v, j}}{N_{3}}-\mu_{3} S_{3} \\
\dot{E}_{3}=\sum_{j=3}^{4} a_{3, j} \beta_{3, j}^{\diamond} S_{3} \frac{I_{v, j}}{N_{3}}-\left(\mu_{3}+\nu_{3}\right) E_{3} \\
\dot{I}_{3}=\nu_{3} E_{3}-\alpha_{3} I_{3} \\
\dot{S}_{v, j}=\Lambda_{v, j}-a_{3, j} \beta_{3, j} S_{v, j} \frac{I_{3}}{N_{3}}-\left(\mu_{v, j}+\delta_{v, j}\right) S_{v, j} \\
\dot{I}_{v, j}=a_{3, j} \beta_{3, j} S_{v, j} \frac{I_{3}}{N_{3}}-\left(\mu_{v, j}+\delta_{v, j}\right) I_{v, j}
\end{array}\right.
$$

for $j=3,4$, since $I_{v, 1}^{0}=0$ (the infected vector at equilibrium from $\mathcal{C}_{1}$ ). The basic reproduction number of Model $\left(C_{2}\right)$ is:

$$
\mathcal{R}_{0, \mathcal{C}_{2}}^{2}=\frac{\nu_{3}}{\alpha_{3}\left(\mu_{3}+\nu_{3}\right) N_{3}}\left[\frac{a_{3,3}^{2} \beta_{3,3} \beta_{3,3}^{\diamond} N_{v, 3}}{\delta_{v, 3}+\mu_{v, 3}}+\frac{a_{3,4}^{2} \beta_{3,4} \beta_{3,4}^{\diamond} N_{v, 4}}{\delta_{v, 4}+\mu_{v, 4}}\right]
$$

The dynamics of System $\left(C_{2}\right)$ is completely determined by $\mathcal{R}_{0, \mathcal{C}_{2}}^{2}$, per Theorem 3.3. For our illustration, we choose parameters such that $\mathcal{R}_{0, \mathcal{C}_{2}}^{2}>1$. That is, a unique strongly endemic equilibrium

$$
\overline{\boldsymbol{x}}_{2}=\left(\bar{S}_{3}, \bar{E}_{3}, \bar{I}_{3}, \bar{S}_{v, 3}, \bar{S}_{v, 4}, \bar{I}_{v, 3}, \bar{I}_{v, 4}\right)
$$

exists and is GAS. Hence, by applying our reduction method on Figure 5, the only irreducible complement left on the network is $\mathcal{C}_{3}$, which is dealt in the next point. 
- On $\mathcal{C}_{3}$, the dynamics of disease is captured by the system:

$$
\left\{\begin{array}{l}
\dot{S}_{i}=\Lambda_{i}-a_{i, 5} \beta_{i, 5}^{\diamond} S_{i} \frac{I_{v, 5}}{N_{i}}-\mu_{i} S_{i} \\
\dot{E}_{i}=a_{i, 5} \beta_{i, 5}^{\diamond} S_{i} \frac{I_{v, 5}}{N_{i}}-\left(\mu_{i}+\nu_{i}\right) E_{h} \\
\dot{I}_{i}=\nu_{i} E_{i}-\alpha_{i} I_{i} \\
\dot{S}_{v, 5}=\Lambda_{v, 5}-\sum_{i=4}^{5} a_{i, 5} \beta_{i, 5} S_{v, 5} \frac{I_{i}}{N_{i}}-a_{3,5} \beta_{3,5} S_{v, 5} \frac{\bar{I}_{3}}{N_{3}}-\left(\mu_{v, 5}+\delta_{v, 5}\right) S_{v, 5} \\
\dot{I}_{v, 5}=\sum_{i=4}^{5} a_{i, 5} \beta_{i, 5} S_{v, 5} \frac{I_{i}}{N_{i}}+a_{3,5} \beta_{3,5} S_{v, 5} \frac{\bar{I}_{3}}{N_{3}}-\left(\mu_{v, 5}+\delta_{v, 5}\right) I_{v, 5},
\end{array}\right.
$$

for $i=4,5$. The corresponding basic reproduction number is:

$$
\mathcal{R}_{0, \mathcal{C}_{3}}^{2}=\frac{N_{v, 5}}{\delta_{v, 5}+\mu_{v, 5}}\left[\frac{a_{4,5}^{2} \beta_{4,5} \beta_{4,5}^{\diamond} \nu_{4}}{\alpha_{4}\left(\mu_{4}+\nu_{4}\right) N_{4}}+\frac{a_{5,5}^{2} \beta_{5,5} \beta_{5,5}^{\diamond} \nu_{5}}{\alpha_{5}\left(\mu_{5}+\nu_{5}\right) N_{5}}\right] .
$$

As started earlier, a strongly endemic equilibrium is GAS, regardless of the value of $\mathcal{R}_{0, \mathcal{C}_{3}}^{2}$. We denote this equilibrium by $\overline{\boldsymbol{x}}_{3}=\left(\bar{S}_{4}, \bar{S}_{5}, \bar{E}_{4}, \bar{E}_{5}, \bar{I}_{4}, \bar{I}_{5}, \bar{S}_{v, 5}, \bar{I}_{v, 5}\right)$. This completes the asymptotic study of System (4.1). The overall equilibrium of System (4.1) is

$$
\boldsymbol{x}^{*}=\left(\boldsymbol{x}_{1}^{0}, \overline{\boldsymbol{x}}_{2}, \overline{\boldsymbol{x}}_{3}, \overline{\boldsymbol{x}}_{4}, \overline{\boldsymbol{x}}_{5}\right),
$$

which is a mixed endemic equilibrium and is GAS.

To summarize, Figure 6 provides the different scenarios that would lead to different types of equilibria.

Remark 4.2. After the first iteration of the method, the local reproduction number on each irreducible components, $\mathcal{R}_{0, \mathcal{C}_{q}}^{2}$, may not always determine the outcome of the infection in that particular component. Indeed, that was the case with $\mathcal{R}_{0, \mathcal{C}_{3}}^{2}$ and $\mathcal{R}_{0, \mathcal{C}_{4}}^{2}$, where they may be below one while the disease is still endemic in their respective irreducible components.

The green path in Figure 6 showcases the case where $\mathcal{R}_{0, \mathcal{C}_{1}}^{2}>1$ and $\mathcal{R}_{0, \mathcal{C}_{5}}^{2}>1$. In this occurrence, the other basic reproduction numbers of other local population are irrelevant and the disease will persist in all populations. Identifying these type of scenarios, allows to allocated the limited resources where it matters the most. For instance, in this particular case, targeting populations of $\mathcal{C}_{2}, \mathcal{C}_{3}$ or $\mathcal{C}_{5}$ would have been a fruitless effort in mitigating or eliminating the infection. Similarly, if $\mathcal{R}_{0, \mathcal{C}_{1}}^{2}>1$ and $\mathcal{R}_{0, \mathcal{C}_{5}}^{2}<1$, the basic reproduction numbers of other local populations in $\mathcal{C}_{2}$ and $\mathcal{C}_{5}$ are irrelevant and the disease will persist in these components. Only $\mathcal{R}_{0, \mathcal{C}_{4}}^{2}$ matters in terms of the overall outcome - red-dashed paths if $\mathcal{R}_{0, \mathcal{C}_{4}}^{2} \leq 1$ and blue-dashed paths if $\mathcal{R}_{0, \mathcal{C}_{4}}^{2}>1$.

\subsection{Numerical simulations}

As highlighted in the Section 4.1, if the network connectivity is reducible, even a relatively low dimensional system may lead to a rich overall dynamical behavior. Depending on the basic reproduction numbers of some strongly connected components, the globally asymptotically stable equilibrium for the system may be a disease-free, a boundary or an interior equilibrium, as seen in Figure 6. In the following, we run some numerical simulations to illustrate the existence of a globally asymptotically stable boundary equilibrium, when the network connectivity of the subpopulations is reducible. To that end, we consider the case studied in Section 4.1, with seven host and seven vector subpopulations. Unless otherwise stated, the baseline parameters of the model 


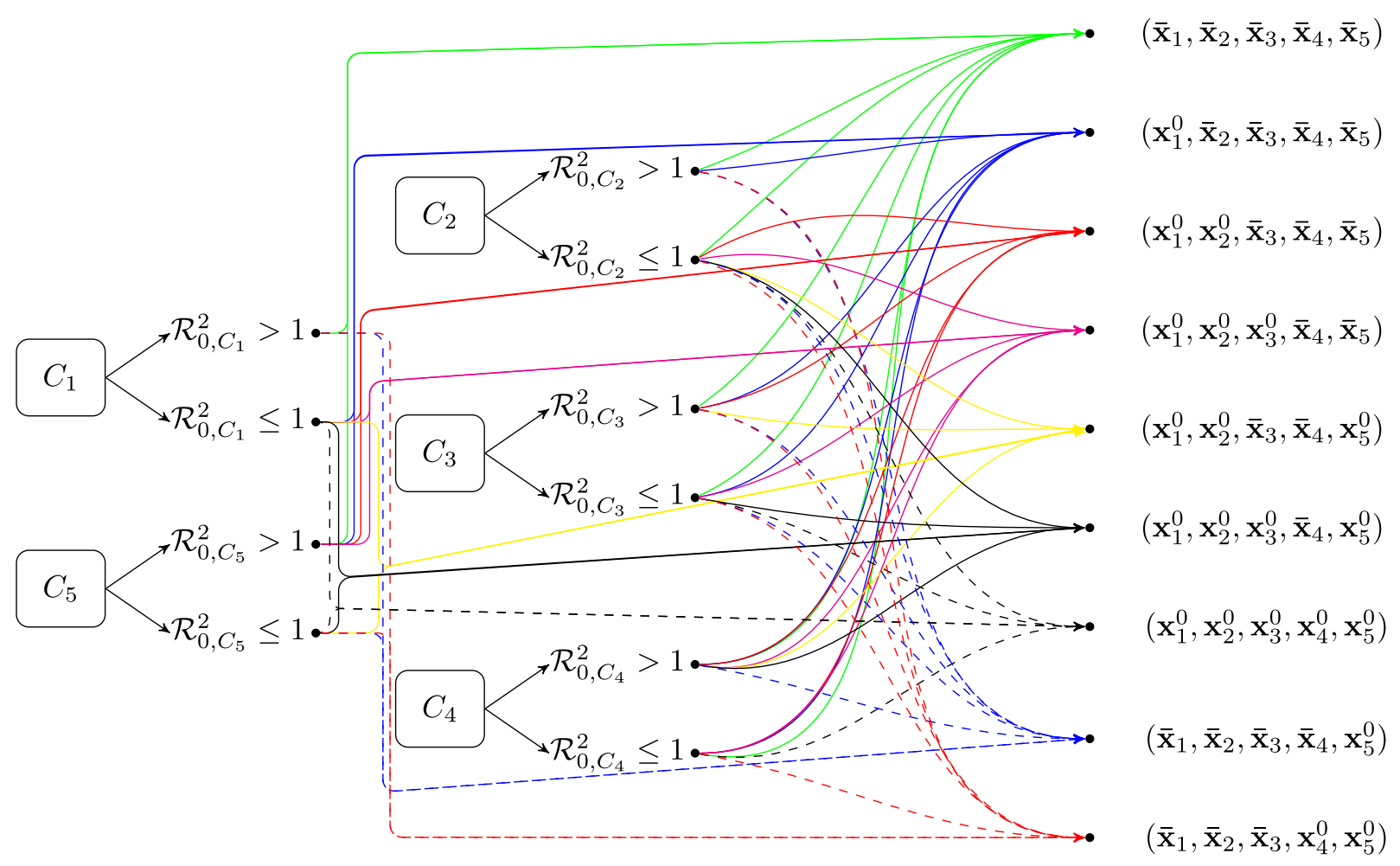

Figure 6. Effects of local basic reproduction numbers on the nature of the equilibria of the overall system.

are chosen as follows, with the corresponding units:

$$
\begin{gathered}
B^{\diamond}=\left(\begin{array}{ccccccc}
0.01 & 0 & 0 & 0 & 0 & 0 & 0 \\
0.03 & 0.01 & 0 & 0 & 0 & 0 & 0 \\
0.01 & 0 & 0.01 & 0.01 & 0 & 0 & 0 \\
0 & 0 & 0 & 0 & 0.01 & 0 & 0 \\
0 & 0 & 0 & 0 & 0.01 & 0 & 0 \\
0 & 0 & 0 & 0 & 0 & 0.01 & 0 \\
0 & 0 & 0 & 0 & 0 & 0 & 0.05
\end{array}\right), B=\left(\begin{array}{ccccccc}
0.02 & 0 & 0 & 0 & 0 & 0.4 & 0 \\
0.01 & 0.01 & 0 & 0 & 0 & 0 & 0 \\
0 & 0 & 0.01 & 0.01 & 0 & 0 & 0 \\
0 & 0 & 0 & 0 & 0.01 & 0 & 0 \\
0 & 0 & 0 & 0 & 0.01 & 0 & 0 \\
0 & 0 & 0 & 0 & 0 & 0.01 & 0 \\
0 & 0 & 0 & 0 & 0 & 0.01 & 0.05
\end{array}\right) \\
\Lambda_{h}=\left(\begin{array}{l}
2 \\
3 \\
1 \\
1 \\
3 \\
1
\end{array}\right), \mu=\frac{1}{1500}\left(\begin{array}{l}
1 \\
1 \\
1 \\
1 \\
1 \\
1 \\
1
\end{array}\right), \nu=\frac{1}{15}\left(\begin{array}{l}
1 \\
1 \\
5 \\
5 \\
5 \\
5 \\
5
\end{array}\right), \alpha=\left(\begin{array}{c}
31 / 1500 \\
31 / 1500 \\
7 / 6000 \\
1 / 600 \\
1 / 375 \\
2 / 1875 \\
11 / 1500
\end{array}\right), \Lambda_{v}=\left(\begin{array}{c}
500 \\
1000 \\
3000 \\
1000 \\
1000 \\
200 \\
1000
\end{array}\right),
\end{gathered}
$$




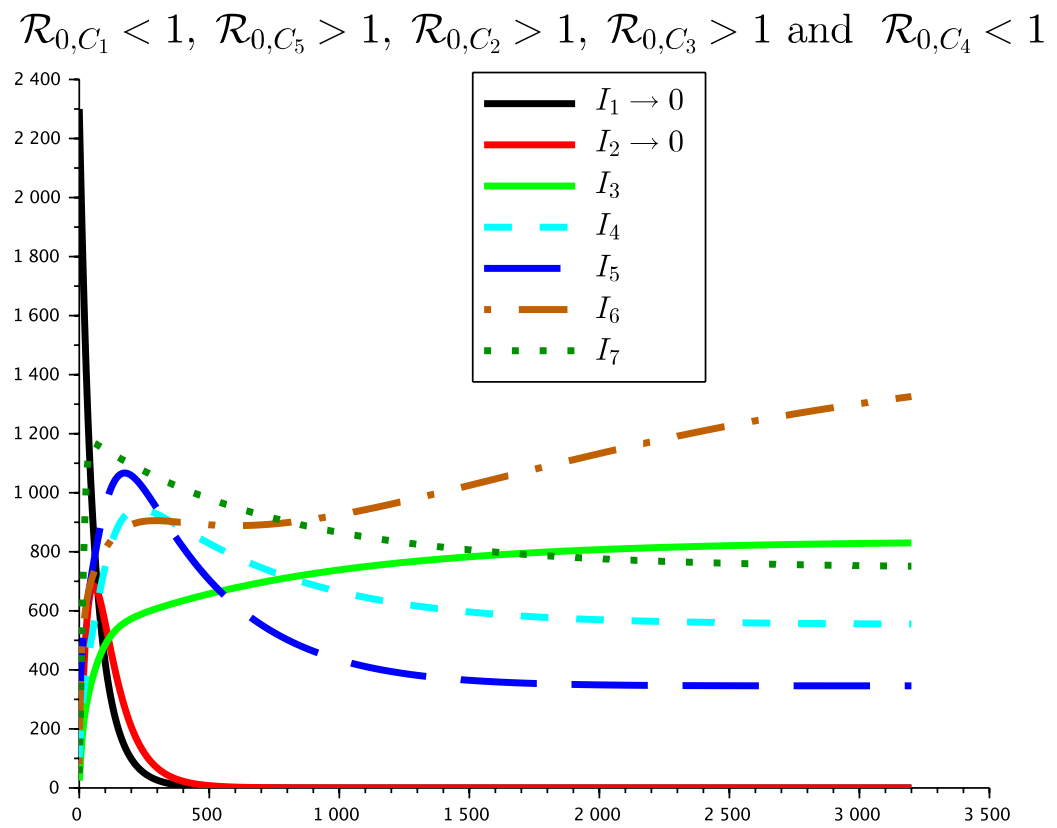

FIGURE 7. Simulation of the dynamics of the infectious host populations.

$$
\mu_{v}=\frac{1}{15}\left(\begin{array}{c}
1 \\
1 \\
1 \\
1 \\
1 \\
1 \\
1
\end{array}\right), \delta_{v}=10^{-4}\left(\begin{array}{c}
10 \\
10 \\
1 \\
7 \\
3 \\
4 \\
5
\end{array}\right) \quad \text { and } \quad a_{i j}=1, \quad \text { for all } i, j \in\{1,2, \ldots, 7\}
$$

With these values, and the expressions of the basic reproduction numbers obtained in Section 4.1, we obtain the following inequalities: $\mathcal{R}_{0, \mathcal{C}_{1}}<1, \mathcal{R}_{0, \mathcal{C}_{5}}>1, \mathcal{R}_{0, \mathcal{C}_{2}}>1, \mathcal{R}_{0, \mathcal{C}_{3}}>1$ and $\mathcal{R}_{0, \mathcal{C}_{4}}<1$. Figures 7 and 8 show the trajectories of the solutions representing the infected host and vector subpopulations, respectively. As predicted by the analysis done above, the solutions converge to a boundary endemic equilibrium. It can be noticed that the disease is endemic in the component $\mathcal{C}_{4}\left(I_{6}(t) \rightarrow \bar{I}_{6}>0\right.$ and $\left.I_{v_{6}}(t) \rightarrow \bar{I}_{v_{6}}>0\right)$ despite the fact that $\mathcal{R}_{0, \mathcal{C}_{4}}<1$. In this case, the mixed-equilibrium obtained coincides with the type of equilibrium obtained in the study case. Indeed, with these conditions on the basic reproduction numbers, the method leads to the second equilibrium from the top on Figure 6: $\boldsymbol{x}^{*}=\left(\boldsymbol{x}_{1}^{0}, \overline{\boldsymbol{x}}_{2}, \overline{\boldsymbol{x}}_{3}, \overline{\boldsymbol{x}}_{4}, \overline{\boldsymbol{x}}_{5}\right)$.

\section{CONCLUSiON}

In this paper, we presented a procedure that provides a complete analysis of a class of large dynamical systems that model a disease that involves the interactions of multiple populations. Indeed, typically the dynamics of an epidemic models that incorporates multiple populations is expressed in terms sharp threshold property. That is, a trivial equilibrium, or the disease-free equilibrium is globally asymptotically stable (GAS) and a unique interior equilibrium exists and is GAS when the basic reproduction number is less or greater than unity, respectively. However, all of these results, particularly that of the stability of the endemic equilibrium, required an irreducibility of the matrix that connects the populations. Here, we provided a method that characterizes the global dynamics of these types of model regardless of the nature of the network configuration. Particularly, 


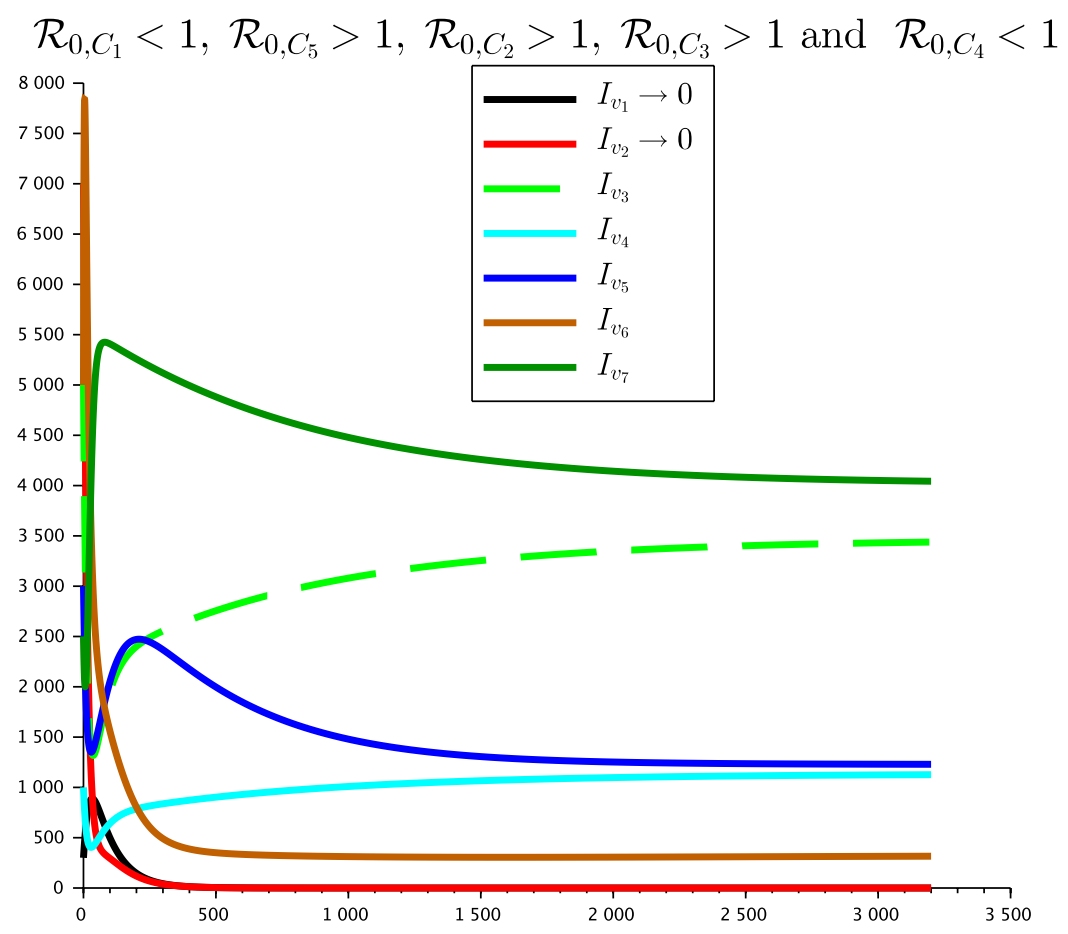

FIGURE 8. Simulation of the dynamics of the infectious vector populations.

we show that the dynamics of the these weakly connected subpopulations is tied to the dynamics of auxiliary systems and the dynamics when the network is irreducible. As a template, we considered the dynamics of two mathematical models that describe the interaction between multiple subpopulations, for the global stability of equilibria are known if the connectivity matrices are irreducible. For these two classes of model, we analyze the asymptotic behavior of the the corresponding auxiliary systems, there by completely characterizing the overall dynamics of these models. The method consists of decomposing the network into a collection of irreducible sub-networks and investigate the dynamics of an auxiliary dynamical system witch accounts for the dynamics on each irreducible subnetwork as well as the effects of the other subnetworks connected to the latter.

More importantly, we presented the procedure along with the natural links between the "local" basic reproduction numbers and the dynamics of the subnetwork with which they may be connected but not strongly connected. The method allows also to pinpoint whether a subnetwork constitutes a source - where the infection not only persists, but also triggers infection in other subnetworks- or a sink -where the infection is maintained only because of its connectivity with other sources. The method consists of two main steps. The first step studies the dynamics of the model on one irreducible component of network, which is a modified system from that of when the network is decoupled from the rest. This auxiliary system is the reduced system on one irreducible subnetwork. The second step consists of using a Vidyasagar's result on triangular dynamical systems to reduce the overall system. By successive incrementation, we complete the analysis of the system. By our method, it is possible to show an interior equilibrium could be GAS even the connectivity matrix is reducible. Moreover, it is also possible to show a boundary equilibrium is also GAS. We applied the method on two complex epidemic model with multiple subpopulations (Sect. 4.1). In these models, we derived the complete dynamics of the corresponding auxiliary systems and deduce the overall dynamics of the initial systems, regardless of the structure of the connectivity between these subpopulations. These results characterizes the overall global dynamics of all types of equilibria. Although a similar result has been presented in [16] and applied in the context of SEIR multi-group models for directly transmitted diseases, our method is simpler to implement. 


\section{ApPENDiX A.}

In this appendix, we briefly introduce some basic elements of graph theory used in the manuscript in order to make it as self-contained as possible.

Definition A.1. A graph is a pair $\mathcal{G}=(\mathcal{V}, \mathcal{E})$ that consists of a set of vertices (or nodes or points) $\mathcal{V}$ and a set of edges (or lines) $\mathcal{E} \subseteq \mathcal{V} \times \mathcal{V}$ :

- Vertices: $v_{i} \in \mathcal{V}$

- Edges: $e_{i j}=\left(v_{i}, v_{j}\right) \in \mathcal{E}$.

For instance, in Figure A.1a, we have:

$\mathcal{V}=\left\{v_{1}, v_{2}, v_{3}, v_{4}\right\}$ and $\mathcal{E}=\left\{\left(v_{1}, v_{2}\right),\left(v_{2}, v_{1}\right),\left(v_{2}, v_{4}\right),\left(v_{4}, v_{2}\right),\left(v_{3}, v_{4}\right),\left(v_{3}, v_{1}\right)\right\}$. A graph $\mathcal{G}$ is called undirected if $e_{i j} \in \mathcal{E} \Longrightarrow e_{j i} \in \mathcal{E}$.

A path is a sequence of edges $\left(v_{i}, v_{l_{1}}\right),\left(v_{l_{1}}, v_{l_{2}}\right), \ldots,\left(v_{l_{r-1}}, v_{j}\right)$, which leads form $v_{i}$ to $v_{j}$. An undirected graph $\mathcal{G}$ is called connected if there is a path between any two distinct nodes of $\mathcal{G}$.

\section{Definition A.2.}

- A directed graph (digraph) $\mathcal{G}$ is strongly connected if for any ordered pairs $\left(v_{i}, v_{j}\right)$ of vertices of $\mathcal{G}$, there exists a path which leads from $v_{i}$ to $v_{j}$.

- A digraph is called weakly connected if there exists an undirected path between any two distinct vertices of $\mathcal{G}$.

A graph can have a matrix representation and the structure of a graph can be determined using the properties of the corresponding matrix. A graph $\mathcal{G}(\boldsymbol{A})$ of $\boldsymbol{A}$ is defined to be the directed graph on $n$ vertices $\left\{v_{1}, v_{2}, \ldots, v_{n}\right\}$ in which there is a directed edge leading from $v_{i}$ to $v_{j}$ if an only if $a_{i j} \neq 0$. For instance, the matrices $\boldsymbol{A}$ and $\boldsymbol{B}$ that represent the graphs $\mathcal{G}(\boldsymbol{A})$ in Figure A.1a and $\mathcal{G}(\boldsymbol{B})$ in Figure A.1b are:

$$
\boldsymbol{A}=\left(\begin{array}{llll}
0 & 2 & 0 & 0 \\
3 & 0 & 0 & 4 \\
6 & 0 & 8 & 1 \\
0 & 5 & 0 & 0
\end{array}\right) \quad \text { and } \quad \boldsymbol{B}=\left(\begin{array}{cccc}
0 & 2 & 7 & 0 \\
3 & 0 & 0 & 4 \\
6 & 0 & 8 & 1 \\
0 & 5 & 0 & 0
\end{array}\right)
$$

Definition A.3. A matrix $\boldsymbol{A}_{n \times n}$ is said to be reducible if there exists a permutation matrix $\boldsymbol{P}$ such that

$$
\boldsymbol{P}^{T} \boldsymbol{A P}=\left(\begin{array}{cc}
\boldsymbol{X} & \boldsymbol{Y} \\
\mathbf{0} & \boldsymbol{Z}
\end{array}\right)
$$

where $\boldsymbol{X}$ and $\boldsymbol{Z}$ are both square. Otherwise $\boldsymbol{A}$ is said to be irreducible.

The irreducibility of matrices can be characterized using associated graphs that represent the corresponding matrices.

The following theorem links the irreducibility of matrix to the connectedness of its graph. Its proof can be found in any standard textbook on matrix analysis (see [22], for example).

Theorem A.4. An $n \times n$ matrix $A$ is irreducible if and only if the corresponding directed graph $\mathcal{G}(\boldsymbol{A})$ is strongly connected.

For example, the matrix $A$ that represents the graphs $\mathcal{G}(\boldsymbol{A})$ in Figure A.1a is reducible, whereas the matrix $B$ that represents the graphs $\mathcal{G}(\boldsymbol{B})$ in Figure A.1b is irreducible.

Per this theorem, if a matrix $A$ is irreducible then its corresponding directed graph $\mathcal{G}(\boldsymbol{A})$ is strongly connected. However, if $\mathcal{G}(\boldsymbol{A})=(\mathcal{V}, \mathcal{E})$ is not strongly connected, it is always possible to partition $\mathcal{V}$ into a disjoint sets of vertices called strongly connected components of the graph. 


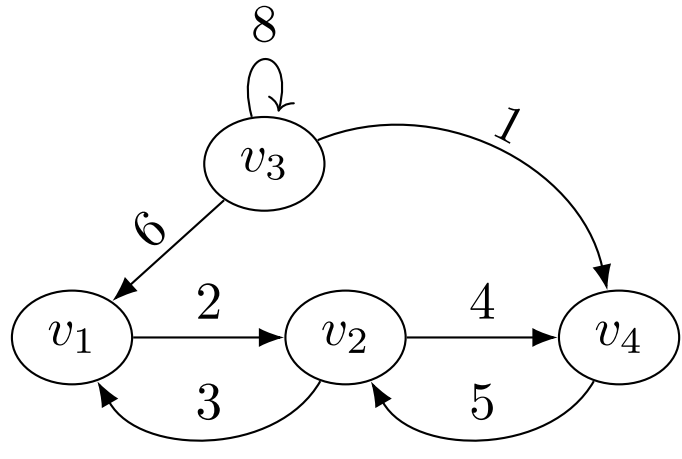

(A)

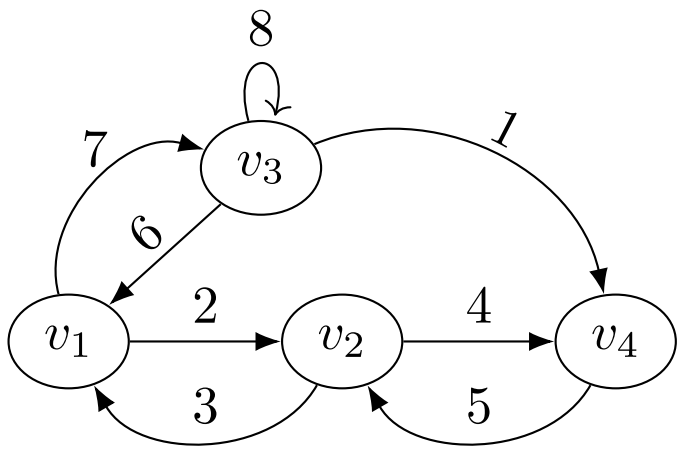

(B)

Figure A.1. Examples of graphs.

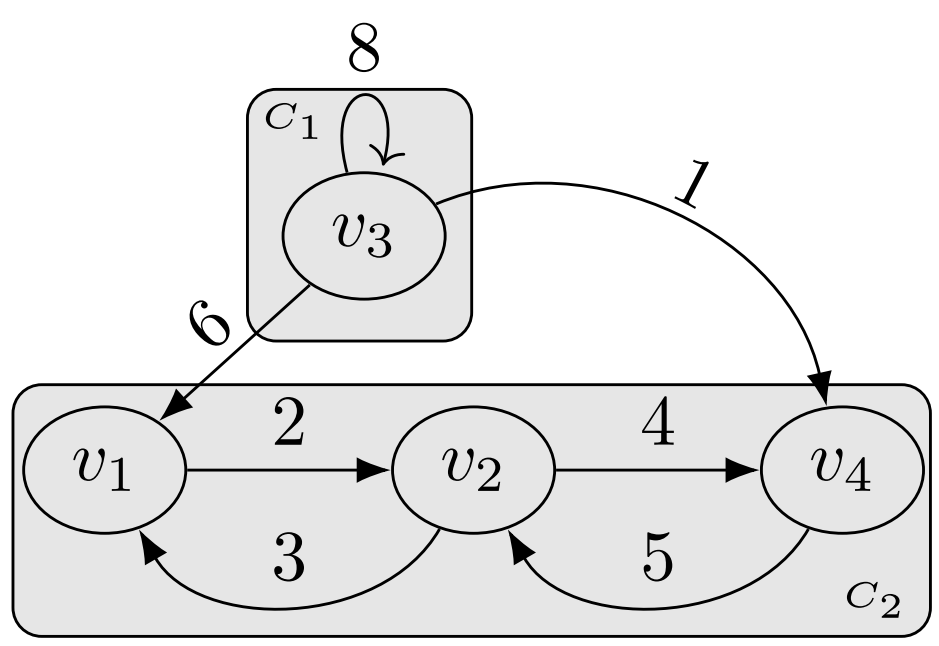

(A) Irreducible components of the graph in Figure A.1a.

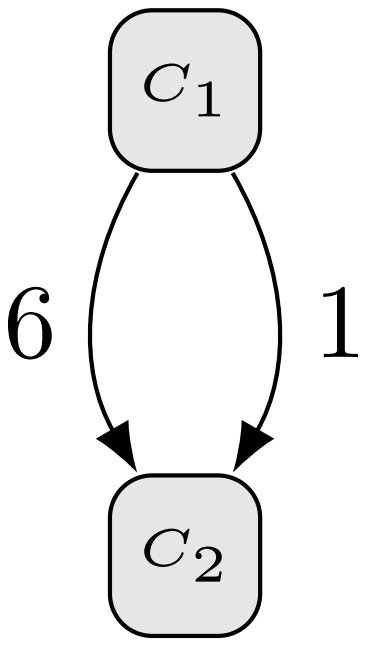

(B) Condensed graph of Figure A.2a.

Figure A.2. Decomposition of non strongly connected graph into strongly connected components.

Definition A.5. A strongly connected component of a directed graph $\mathcal{G}(\boldsymbol{A})=(\mathcal{V}, \mathcal{E})$ is the maximal set of vertices $\mathcal{C} \subseteq \mathcal{V}$ such that for any ordered pairs $\left(v_{i}, v_{j}\right)$ of vertices of $\mathcal{C}$, there exists a path which leads from $v_{i}$ to $v_{j}$.

For instance, the graph $\mathcal{G}(\boldsymbol{A})$ in Figure A.1a is not strongly connected as its corresponding matrix is not irreducible. However, it is possible to decompose $\mathcal{G}(\boldsymbol{A})$ into a set of irreducible components as shown in Figure A.2. Particularly, Figure A.2a decompose the vertices of Figure A.1a into two irreducible components $\mathcal{C}_{1}$ and $\mathcal{C}_{2}$ (shaded sets of vertices). Figure A.2b shows the condensed graph in terms of the irreducible components. The irreducible component $\mathcal{C}_{1}$ is called source (a node without incoming edges) and $\mathcal{C}_{2}$ is called sink (a node without outgoing edges).

Acknowledgements. We are grateful to anonymous referees for valuable comments and suggestions that led to an improvement of this paper. 


\section{REFERENCES}

[1] R.M. Anderson and R.M. May, Infectious Diseases of Humans. Dynamics and Control. Oxford Science Publications (1991).

[2] J. Arino, Diseases in metapopulations, in Modeling and Dynamics of Infectious Diseases, edited by Z. Ma, Y. Zhou, and J. Wu. Volume 11 of Series in Contemporary Applied Mathematics. World Scientific (2009) 65-123. Also CDM Preprint Series report 2008-04.

[3] J. Arino and P. van den Driessche, Disease spread in metapopulations, in Nonlinear dynamics and evolution equations, edited by X.-O Zhao and X. Zou. Vol. 48 of Fields Institute Communications. AMS, Providence, R.I. (2006) 1-13.

[4] J. Arino, A. Ducrot and P. Zongo, A metapopulation model for malaria with transmission-blocking partial immunity in hosts. J. Math. Biol. 64 (2012) 423-448.

[5] P. Auger, E. Kouokam, G. Sallet, M. Tchuente and B. Tsanou, The Ross-Macdonald model in a patchy environment. Math. Biosci. 216 (2008) 123-131.

[6] A. Berman and R.J. Plemmons, Nonnegative matrices in the mathematical sciences. SIAM (1994).

[7] D.M. Bichara, Effects of migration on vector-borne diseases with forward and backward stage progression. Discr. Continu. Dyn. Syst. Ser. B 24 (2019) 6297-6323.

[8] D.M. Bichara, Global analysis of multi-host and multi-vector epidemic models. J. Math. Anal. Appl. 475 (2019) $1532-1553$.

[9] D.M. Bichara and A. Iggidr, Multi-patch and multi-group epidemic models: a new framework. J. Math. Biol. 77 (2018) 107-134.

[10] D.M. Bichara, Y. Kang, C. Castillo-Chavez, R. Horan and C. Perrings, Sis and sir epidemic models under virtual dispersal. Bull. Math. Biol. 77 (2015) 2004-2034.

[11] D.M. Bichara and C. Castillo-Chavez, Vector-borne diseases models with residence times - a Lagrangian perspective. Math. Biosci. 281 (2016) 128-138.

[12] B. Bonzi, A. Fall, A. Iggidr and G. Sallet, Stability of differential susceptibility and infectivity epidemic models. J. Math. Biol. 62 (2011) 39-64.

[13] C. Cosner, J.C. Beier, R.S. Cantrell, D. Impoinvil, L. Kapitanski, M.D. Potts, A. Troyo and S. Ruan, The effects of human movement on the persistence of vector-borne diseases. J. Theor. Biol. 258 (2009) 550-560.

[14] G. Cruz-Pacheco, L. Esteva and C. Vargas, Multi-species interactions in West Nile virus infection. J. Biol. Dyn. 6 (2012) $281-298$.

[15] O. Diekmann, J.A.P. Heesterbeek and J.A.J. Metz, On the definition and the computation of the basic reproduction ratio $R_{0}$ in models for infectious diseases in heterogeneous populations. J. Math. Biol. 28 (1990) 365-382.

[16] P. Du and M.Y. Li, Impact of network connectivity on the synchronization and global dynamics of coupled systems of differential equations. Physica D 286 (2014) 32-42.

[17] A. Fenton, D.G. Streicker, O.L. Petchey and A.B Pedersen, Are all hosts created equal? Partitioning host species contributions to parasite persistence in multihost communities. Am. Nat. 186 (2015) 610-622.

[18] A.K. Githeko, J.M. Ayisi, P.K. Odada, F.K. Atieli, B.A. Ndenga, J.I. Githure and G. Yan, Topography and malaria transmission heterogeneity in western Kenya highlands: prospects for focal vector control. Malaria J. 5 (2006).

[19] H. Guo, M.Y. Li and Z. Shuai, Global stability of the endemic equilibrium of multigroup SIR epidemic models. Can. Appl. Math. Quart. 14 (2006) 259-284.

[20] H. Guo, M.Y. Li and Z. Shuai, A graph-theoretic approach to the method of global Lyapunov functions. Proc. Am. Math. Soc. 136 (2008) 2793-2802.

[21] I. Hanski, Metapopulation Ecology. Oxford University Press (1999).

[22] R.A. Horn and C.R. Johnson, Matrix analysis. Cambridge University Press (1985).

[23] A. Iggidr, G. Sallet and B. Tsanou, Global stability analysis of a metapopulation SIS epidemic model. Math. Popul. Stud. 19 (2012) 115-129.

[24] A. Iggidr, G. Sallet and M.O. Souza, On the dynamics of a class of multi-group models for vector-borne diseases. J. Math. Anal. Appl. 2 (2016) 723-743.

[25] W.B. Karesh, A. Dobson, J.O. Lloyd-Smith, J. Lubroth, M.A. Dixon, M. Bennett, S. Aldrich, T. Harrington, P. Formenty, E.H. Loh and C.C. Machalaba, Ecology of zoonoses: natural and unnatural histories. The Lancet 380 (2012) $1936-1945$.

[26] W.O. Kermack and A.G. McKendrick, Contributions to the mathematical theory of epidemics-I. 1927. Bull. Math. Biol. 53 (1991) 33-55.

[27] A. Lajmanovich and J.A. Yorke, A deterministic model for gonorrhea in a nonhomogeneous population. Math. Biosci. 28 (1976) 221-236.

[28] D.J. Rodríguez and L. Torres-Sorando, Models of infectious diseases in spatially heterogeneous environments. Bull. Math. Biol. 63 (2001) 547-571.

[29] N.W. Ruktanonchai, D.L. Smith and P. De Leenheer, Parasite sources and sinks in a patched Ross-MacDonald malaria model with human and mosquito movement: implications for control. Math. Biosci. 279 (2016) 90-101.

[30] M. Salmani and P. van den Driessche, A model for disease transmission in a patchy environment. Discr. Continu. Dyn. Syst. B 6 (2006) 185-202.

[31] D.L. Smith, J. Dushoff and F. Ellis McKenzie, The risk of a mosquito-borne infection in a heterogeneous environment. PLoS Biol. 2 (2004) 1957-1964.

[32] H.L. Smith, Monotone dynamical systems. An introduction to the theory of competitive and cooperativ systems. AMS, Providence, R.I. (1995). 
[33] P. van den Driessche and J. Watmough, Reproduction numbers and sub-threshold endemic equilibria for compartmental models of disease transmission. Math. Biosci. 180 (2002) 29-48.

[34] P. van den Driessche, Y.-H Hsieh and L. Wang, Impact of travel between patches for spatial spread of disease. Bull. Math. Biol. 69 (2007) 1355-1375.

[35] M. Vidyasagar, Decomposition techniques for large-scale systems with nonadditive interactions: stability and stabilizability. IEEE Trans. Autom. Control 25 (1980) 773-779.

[36] J.P. Webster, A. Borlase and J.W. Rudge, Who acquires infection from whom and how? disentangling multi-host and multimode transmission dynamics in the 'elimination' era. Philos. Trans. Roy. Soc. B 372 (2017).

[37] World Bank, People, pathogens and our planet, volume 1: Towards a one health approach for controlling zoonotic diseases. World Bank, Agriculture and Rural Development Health, Nutrition and Population (2010). 